\title{
Stability of Ordinary Differential Equations with Colored Noise Forcing
}

\author{
Timothy Blass $^{1} \quad$ L.A. Romero ${ }^{2}$
}

August 15, 2018

\begin{abstract}
We present a perturbation method for determining the moment stability of linear ordinary differential equations with parametric forcing by colored noise. In particular, the forcing arises from passing white noise through an $n$th order filter. We carry out a perturbation analysis based on a small parameter $\varepsilon$ that gives the amplitude of the forcing. Our perturbation analysis is based on a ladder operator approach to the vector Ornstein-Uhlenbeck process. We can carry out our perturbation expansion to any order in $\varepsilon$, for a large class linear filters, and for quite arbitrary linear systems. As an example we apply our results to the stochastically forced Mathieu equation.
\end{abstract}

Subject Class: Primary: 93E15; Secondary: 60H10, 34D10.

\section{Introduction}

\subsection{A Class of Stochastically Forced Linear Equations}

The original goal of this work was to develop a framework for analyzing the stability of the stochastically forced Mathieu equation:

$$
\ddot{x}+\gamma \dot{x}+\left(\omega_{0}^{2}+\varepsilon f(t)\right) x=0,
$$

where $f$ is a stochastic process, and the stability is determined by the boundedness of the second moment $\left\langle\left\langle x^{2}(t)\right\rangle\right\rangle[5,13$. Here, $\langle\langle\cdot\rangle\rangle$ denotes the sampleaverage. We wanted to avoid heuristic methods, and consider cases where $f(t)$ is a stochastic process with a realistic power spectral density. In particular,

\footnotetext{
${ }^{1}$ Department of Mathematical Sciences, Carnegie Mellon University, Pittsburgh, PA 15213 , USA tblass@andrew.cmu.edu

${ }^{2}$ Computational Mathematics and Algorithms Department, Sandia National Laboratories, MS 1320, P.O. Box 5800, Albuquerque, NM 87123-1320, Iromero@sandia.gov

${ }^{3}$ Sandia National Laboratories is a multi-program laboratory managed and operated by Sandia Corporation, a wholly owned subsidiary of Lockheed Martin Corporation, for the U.S. Department of Energy's National Nuclear Security Administration under contract DE-AC0494AL85000.
} 
we do not want to assume that $f$ is white noise. Hence we want to analyze the case where $f(t)$ is colored noise. However, in order to rigorously derive a Fokker-Planck equation for a stochastic differential equation, the governing equation must include only white noise [5. We can achieve both goals of rigor and realistic power spectral density by letting $f$ be the output of a linear filter that is forced by a vector white noise $\boldsymbol{\xi}$. That is,

$$
\begin{aligned}
& \dot{\mathbf{s}}=\mathbf{H} \mathbf{s}+\boldsymbol{\xi}(t), \\
& f(t)=\langle\mathbf{a}, \mathbf{s}(t)\rangle,
\end{aligned}
$$

where $\mathbf{H}$ is an $n \times n$ real, diagonalizable matrix, whose eigenvalues have negative real parts, a $\in \mathbb{R}^{n}$, and $\langle\cdot, \cdot\rangle$ is the standard inner product on $\mathbb{C}^{n}$. We will take deterministic initial condition $\mathbf{s}(0)=\mathbf{0}$. We assume the noise vector $\boldsymbol{\xi}$ is weighted white noise, meaning

$$
\langle\langle\boldsymbol{\xi}\rangle\rangle=0, \quad\left\langle\left\langle\boldsymbol{\xi}(t+\tau) \boldsymbol{\xi}^{T}(t)\right\rangle\right\rangle=\mathbf{B} \delta(\tau),
$$

where $\mathbf{B}$ is symmetric and positive semi-definite. Thus, when $\mathbf{s}(t)$ solves (2) it is a standard vector-valued Ornstein-Uhlenbeck process. We refer to the scalar process, $f(t)=\langle\mathbf{a}, \mathbf{s}(t)\rangle$, as colored noise or as an $n$ th-order filter provided $\mathbf{s}(t)$ solves (2). We will make only mild requirements on the matrices $\mathbf{H}$ and $\mathbf{B}$, thereby allowing for wide variability in the power spectral density of the resulting process $\langle\mathbf{a}, \mathbf{s}(t)\rangle$. Thus, in allowing for a wide range of choices of $\mathbf{H}, \mathbf{B}$, and $\mathbf{a}$, our approach accommodates a broad class of colored noise forcing terms.

In this paper we will be concerned with the more general problem of linear equations that are being parametrically forced by the function $f(t)$ in equation (31). That is equations of the form

$$
\dot{\mathbf{x}}=\mathbf{A}_{0} \mathbf{x}+\varepsilon\langle\mathbf{a}, \mathbf{s}\rangle \mathbf{A}_{1} \mathbf{x},
$$

where $\mathbf{s}$ is the solution to the stochastic equation (2), $\mathbf{x}(t) \in \mathbb{R}^{N}$ for some $N \geq 1$, and $\mathbf{A}_{0}, \mathbf{A}_{1}$ are $N \times N$ constant matrices.

The purpose of this paper is to present a perturbation method (assuming $\varepsilon$ is small) for determining the stability of the solution $\mathbf{x}(t)$ of (5), by which we mean the boundedness of the second moments of $\mathbf{x}(t)$. However, our method applies to the $p$ th moment, so we will not limit our analysis to second moments only. Van Kampen has presented a heuristic approach to the case of colored noise forcing, 24. Though derived by completely different means, his result for the Mathieu equation (10) is the same as ours when considering only the firstmoments, and without damping. He arrives at his result by truncating some series at order $\varepsilon^{2}$, and is only expected to be valid to this order. Our method is rigorous, can be applied to find solutions to any order in $\varepsilon$, and applies to any moment. We discuss this further in $\$ 5.2$,

We were originally interested in equation (11) as a model for the response of capillary gravity waves to a time-varying gravitational field arising from random vertical motions of a container with a free surface (as in [19]). Here $f(t)$ represents the random fluctuations in acceleration. Since the Fourier transform 
of an acceleration should vanish at zero, along with its derivative, the power spectral density of a realistic process $f$ should satisfy $S(0)=S^{\prime}(0)=0$. For example, we can construct a two-dimensional filter using the system (2) that has the power spectral density

$$
S(\omega)=\frac{\sigma \beta^{2} \omega^{2}}{\left(\omega^{2}+\mu_{1}^{2}\right)\left(\omega^{2}+\mu_{2}^{2}\right)},
$$

by choosing

$$
\mathbf{H}=\left(\begin{array}{cc}
-\mu_{1} & 0 \\
\beta & -\mu_{2}
\end{array}\right), \quad \mathbf{B}=\left(\begin{array}{ll}
\sigma & 0 \\
0 & 0
\end{array}\right), \quad \mathbf{a}=\left(\begin{array}{c}
\beta \\
-\mu_{2}
\end{array}\right), \quad \mu_{1}, \mu_{2}, \sigma>0 .
$$

The formula for $S(\omega)$ in equation (6) follows from Corollary 7 in Appendix C.

The stochastically forced Mathieu equation has been analyzed before, for instance in 2, 3, 4, 9, 16, 22, but not for the case (1), or the general setting (5). In 16, 22, they consider additive forcing, and in 2, 3, 4, they consider a different type of parametric forcing. In [9] they consider a different class of colored noises and study stability by truncating an infinite hierarchy of moment equations. Other studies concern Lyapunov stability, or rely on numerical methods. Our analysis applies to a broad class of equations (5) with a wide variety of forcing terms (2)), is semi-analytical (relying only on numerics for the computation of eigenvalues of small matrices), and can be applied to any moment.

\subsection{Ladder Operators and the Vector Ornstein-Uhlenbeck Process}

Our perturbation analysis of the moment stability of equation (5) relies heavily on a simple characterization of the eigenvalues and eigenfunctions of the Fokker-Planck equation associated with equation (2). In particular, in $\$ 2$ and $\$ 3$ we characterize the spectrum using ladder operators by generalizing Dirac's creation and annihilation operator approach to the quantum harmonic oscillator 11. An understanding of the spectrum and eigenfunctions in terms of its ladder operators is crucial to developing the perturbation theory in 4 Though other authors have used ladder operators for Ornstein-Uhlenbeck processes, they have only considered the scalar case $n=1[20,21,23,25]$. We believe the extension to the vector case is by no means trivial, and is interesting in its own right.

The probability density function $P\left(s_{1}, \ldots, s_{n}, t\right)$ associated with the process $\mathbf{s}(t)$ defined by equations (2) and (44) satisfies the Fokker-Planck equation

$$
\partial_{t} P=\mathcal{D} P, \quad \text { with } \quad \mathcal{D} P=\frac{1}{2} \operatorname{div}(\mathbf{B} \nabla P)-\operatorname{div}(\mathbf{H} \mathbf{s} P) .
$$

$\mathcal{D}$ is called the Fokker-Planck operator associated to (2). See [12] for a derivation of this equation. We note that the Fokker-Planck equation (7) is the same in both the Itô and Stratonovich interpretations because the matrix $\mathbf{B}$ is independent of $\mathbf{s}$ (see [12]). The operator $\mathcal{D}$ will play a crucial role in our stability analysis. 
In $₫ 2$ we begin by analyzing the operator $\mathcal{D}$ in terms of its associated ladder operators. That is, operators $\mathcal{L}$ satisfying the commutator equation

$$
[\mathcal{D}, \mathcal{L}]=\mu \mathcal{L}
$$

As in Dirac's theory of the harmonic oscillator, the significance of the ladder operators stems from the fact that if $\phi$ is an eigenfunction of $\mathcal{D}$ with eigenvalue $\lambda$, then the function $\mathcal{L} \phi$ will either vanish, or be an eigenfunction of $\mathcal{D}$ with eigenvalue $\lambda+\mu$.

In $₫ 2$ we show that we can construct the ladder operators by solving a matrix eigenvalue problem

$$
\mathbf{T y}=\mu \mathbf{y}, \quad \mathbf{T}=\mathbf{D A},
$$

where $\mathbf{A}$ is an antisymmetric matrix and $\mathbf{D}$ is a symmetric matrix, expressed in terms of $\mathbf{H}, \mathbf{B}$. We show there are $n$ raising operators, $\mathcal{L}_{k}$, which generate new eigenfunctions of $\mathcal{D}$ with an increase in the real part of the eigenvalue, and $n$ lowering operators $\mathcal{L}_{-k}$ that correspondingly decrease the real part of the eigenvalue. We also show that $\mathcal{D}$ can be expressed in terms of its ladder operators. In particular,

$$
\mathcal{D}=\sum_{k=1}^{n} \mu_{k} \mathcal{L}_{-k} \mathcal{L}_{k} .
$$

where $\mu_{k}$ is the increment of the ladder operator $\mathcal{L}_{k}$. That is, $\left[\mathcal{D}, \mathcal{L}_{k}\right]=\mu_{k} \mathcal{L}_{k}$. This representation is useful for determining the spectrum of $\mathcal{D}$.

In $\$ 3$ we characterize the solutions of

$$
\mathcal{D} \phi=\chi \phi
$$

in terms of the ladder operators, $\mathcal{L}$, and increments, $\mu$, solving (8). In particular, we show that any eigenvalue $\chi$ of $\mathcal{D}$ can be written as

$$
\chi_{\mathbf{k}}=-\sum_{j=1}^{n} k_{j} \mu_{j}
$$

where $\mu_{j}$ are the increments of the ladder operators with positive real parts, and the $k_{j}$ are non-negative integers. We will see that the increments $\mu_{j}$ are the negative of the eigenvalues of the matrix $\mathbf{H}$ defining the filter in equation (3). We also show that any eigenfunction of $\mathcal{D}$ can be obtained by applying the ladder operator to the eigenfunction $\Phi_{0}(\mathbf{s})$ associated with the eigenvalue $\chi=0$ of $\mathcal{D}$, which is the eigenvalue with the largest real part.

The results summarized in the last paragraph rely on the fact that real parts of the eigenvalues of $\mathcal{D}$ are bounded above (see Lemma 6), which is proved in [17, 18, 22, but we give a different and simple proof of this in Appendix B. Here, the domain of $\mathcal{D}$ is the set of functions that have bounded moments of any order. The spectrum and eigenfunctions of $\mathcal{D}$ have been studied before (see [17, 18, 22]) but not in the context of ladder operators. 


\subsection{Perturbation Expansion for Moment Stability Analy- sis}

In $₫ 4$ we use the classical perturbation theory of eigenvalues to carry out an analysis of the stability of equation (5). Our analysis begins by considering the ODEs for $\mathbf{s}(t)$ and $\mathbf{x}(t)$ together as a single ODE system. The probability density function $P\left(s_{1}, \ldots, s_{n}, x_{1}, \ldots, x_{N}, t\right)$ for the combined system (2) and (5) solves the Fokker-Planck equation

$$
\partial_{t} P=\frac{1}{2} \operatorname{div}_{\mathbf{s}}\left(\mathbf{B} \nabla_{\mathbf{s}} P\right)-\operatorname{div}_{\mathbf{s}}(\mathbf{H} \mathbf{s} P)-\operatorname{div}_{\mathbf{x}}\left(\left(\mathbf{A}_{0}+\varepsilon\langle\mathbf{a}, \mathbf{s}\rangle \mathbf{A}_{1}\right) \mathbf{x} P\right)
$$

The notation $\operatorname{div}_{\mathbf{s}}$ and $\nabla_{\mathbf{s}}$ refer to divergence and gradient with respect to only the $s_{j}$ variables, and similarly $\operatorname{div}_{\mathbf{x}}$ is divergence in $\mathbf{x}$ variables. Equation (13) is the same in both the Itô and Stratonovich interpretations because the matrix $\mathbf{B}$ is independent of $\mathbf{s}$ and $\mathbf{x}$ (see [12]).

We can derive an equation for the $p$ th marginal moments by multiplying (13) by monomials $\mathbf{x}^{\alpha}$ and integrating with respect to $d \mathbf{x}$, where $\alpha$ is a multi-index of order $p$. The result is an equation for $\mathbf{m}(\mathbf{s}, t)$, a vector of the $p$ th marginal moments, which is of the form

$$
\partial_{t} \mathbf{m}=\mathcal{D} \mathbf{m}+\boldsymbol{\Gamma}_{0} \mathbf{m}+\varepsilon\langle\mathbf{a}, \mathbf{s}\rangle \boldsymbol{\Gamma}_{1} \mathbf{m}
$$

Note that $\mathcal{D}$ is a differential operator in the $\mathbf{s}$ variables only,

$$
\mathcal{D} \varphi=\frac{1}{2} \operatorname{div}_{\mathbf{s}}\left(\mathbf{B} \nabla_{\mathbf{s}} \varphi\right)-\operatorname{div}_{\mathbf{s}}(\mathbf{H} \mathbf{s} \varphi)
$$

In equation (14) each component of $\mathbf{m}(\mathbf{s}, t)$ is of the form $\int_{\mathbb{R}^{N}} \mathbf{x}^{\alpha} P(\mathbf{x}, \mathbf{s}, t) d \mathbf{x}$ for some multi-index $\alpha$ with $|\alpha|=p$. $\mathcal{D} \mathbf{m}$ indicates $\mathcal{D}$ applied to each component of $\mathbf{m}$. For much of our analysis we can assume that the matrices $\boldsymbol{\Gamma}_{0}$ and $\boldsymbol{\Gamma}_{1}$ are given to us, but we illustrate how to obtain these matrices from the matrices $\mathbf{A}_{0}$ and $\mathbf{A}_{1}$ for the particular case of the Mathieu equation in $\$ 5$. The matrices $\boldsymbol{\Gamma}_{0}, \boldsymbol{\Gamma}_{1}$ in (14) are constant and depend on which moments one is considering (see example in equation (53)). There are $J=\left(\begin{array}{c}N+p-1 \\ p\end{array}\right)$ distinct $p$ th order monomials in $N$ variables, therefore $\boldsymbol{\Gamma}_{0}$ and $\boldsymbol{\Gamma}_{1}$ are $J \times J$ matrices.

As in a standard stability analysis, in order to determine the stability of (14), we look for solutions of the form $\widetilde{\mathbf{m}}(\mathbf{s}, t)=e^{\lambda t} \mathbf{m}(\mathbf{s})$. Our equation for $\mathbf{m}(\mathbf{s})$ becomes

$$
\lambda \mathbf{m}=\mathcal{D} \mathbf{m}+\boldsymbol{\Gamma}_{0} \mathbf{m}+\varepsilon\langle\mathbf{a}, \mathbf{s}\rangle \boldsymbol{\Gamma}_{1} \mathbf{m} .
$$

That is, the equation for the $p$ th marginal moments of $\mathbf{x}(t)$ can be written as an eigenvalue problem, and stability is decided by the sign of the real part of the largest eigenvalue.

We do a perturbation analysis assuming that the magnitude $\varepsilon$ of the forcing is small. Our analysis relies on the fact that that when $\varepsilon=0$ the eigenfunctions of equation (16) are the direct product of the eigenfunctions of $\mathcal{D}$ and the eigenvectors of the matrix $\boldsymbol{\Gamma}_{0}$. 
A key observation (see Lemma 9) for the perturbation analysis is that for any vector a we can determine constants $\alpha_{k}$ and $\beta_{k}$ such that $\langle\mathbf{a}, \mathbf{s}\rangle$ can be written as

$$
\langle\mathbf{a}, \mathbf{s}\rangle=\sum_{k=1}^{n}\left(\alpha_{k} \mathcal{L}_{k}+\beta_{k} \mathcal{L}_{-k}\right),
$$

where $\mathcal{L}_{ \pm k}$ are the ladder operators satisfying (8). The proof of Lemma 9 is given in Appendix D.

In 84 we show that when $\varepsilon=0$, the eigenvalue of equation (16) with the largest real part is the same as the largest eigenvalue of the matrix $\boldsymbol{\Gamma}_{0}$. If $\lambda_{0}$ is this unperturbed eigenvalue, then $\lambda(\varepsilon)=\lambda_{0}+\lambda_{2} \varepsilon^{2}+\ldots$ with

$$
\lambda_{2}=\sum_{j=1}^{J}\left\langle\boldsymbol{\psi}_{1}, \boldsymbol{\Gamma}_{1} \boldsymbol{\phi}_{j}\right\rangle\left\langle\boldsymbol{\psi}_{j}, \boldsymbol{\Gamma}_{1} \boldsymbol{\phi}_{1}\right\rangle G\left(\nu_{1}-\nu_{j}\right)
$$

where $\nu_{1}=\lambda_{0}, \nu_{j}$ are the eigenvalues of $\boldsymbol{\Gamma}_{0}$, and $\boldsymbol{\phi}_{j}, \boldsymbol{\psi}_{j}$ are the eigenvectors and normalized adjoint eigenvectors of $\boldsymbol{\Gamma}_{0}$. Equation (18) uses the extended power spectral density $G(z)$, which is defined for a general stationary random process in (79), and is given explicitly in (81) for the filter $\langle\mathbf{a}, \mathbf{s}(t)\rangle$.

The form of $\lambda_{2}$ in (18) is derived for forcing terms that have the form (3), however, the fact that this is simply a weighted sum of values of $G$, whose coefficients depend only on $\boldsymbol{\Gamma}_{0}, \boldsymbol{\Gamma}_{1}$ (which do not depend on the filter), suggests such a formula could hold for any process with a well-defined extended power spectral density. We have carried the perturbation analysis to higher orders, but the higher-order coefficients do not appear to have such a simple form as in equation (18).

The method in $₫ 4$ involves constructing matrices $\boldsymbol{\Gamma}_{0}$ and $\boldsymbol{\Gamma}_{1}$, which, as mentioned earlier, depend on $\mathbf{A}_{0}, \mathbf{A}_{1}$, and the representation of the $p$ th marginal moments as a vector. We use the stochastic Mathieu equation as a specific example in $\$ 5$ In $\$ 5.3$ we discuss a numerical method for determining the stability of (5) without assuming that $\varepsilon$ is small. We compare these numerical results to our perturbation results up to both second and fourth order for the Mathieu equation, and show they are in excellent agreement. In $\$ 5.4$ we give a second representation (whose derivation is given in [8]) for $\lambda_{2}$ that does not involve the matrices $\boldsymbol{\Gamma}_{0}$ and $\boldsymbol{\Gamma}_{1}$, but deals directly with the matrices $\mathbf{A}_{0}$ and $\mathbf{A}_{1}$. We have found that this representation simplifies numerical computations.

\section{Existence and Properties of Ladder Operators}

In this section we define the notion of a ladder operator, show how to construct these operators, and prove some basic lemmas about them. Lemma 1 shows how ladder operators can be used to generate new eigenfunctions that have their eigenvalue changed by the increment of the ladder operator. Lemma 2 shows how to find the ladder operators $\mathcal{L}_{k}$ and their increments $\mu_{k}$ by solving a matrix eigenvalue problem. Lemma 3 shows that the increments of the ladder operators 
are zero and $\pm \mu_{k}$, where $-\mu_{k}$ are the eigenvalues of the matrix $\mathbf{H}$ defining our filter (see equation (2)). Lemma 4 gives the commutator relations for the ladder operators, and Lemma 5 shows that the operator $\mathcal{D}$ can be expressed as a weighted sum of $\mathcal{L}_{-k} \mathcal{L}_{k}$, where $\mathcal{L}_{k}$ are the ladder operators. Throughout this section (and the rest of the paper) the operator $\mathcal{D}$ is that defined in (15).

The basic lemmas in this section are crucial to the rest of this paper, and hence we have tried to write this section so that the lemmas stand out clearly. Though the lemmas are all easily stated, the proofs of some of the lemmas are quite technical, especially when attention is given to ensuring that they apply for complex eigenvalues of the matrix $\mathbf{T}$. For this reason, we have relegated many of the proofs to Appendix A.

Before discussing ladder operators it should be noted that we define the domain of $\mathcal{D}$ as the set of functions that have bounded moments of any order. Thus, our definition of the domain of $\mathcal{D}$ differs from that given in [17. In that paper they defined the domain based on the exponential decay of the eigenfunction $\Phi_{0}$ that we define in equation (32) and discuss in 93 . The two definitions of the domain give the identical eigenfunctions, but we believe ours is more natural since it does not require knowing the solution ahead of time. In [17] they discuss a continuous spectrum that arises if the domain is defined so that the eigenfunctions of $\mathcal{D}$ are only required to be square integrable (or some similarly less restrictive condition). An examination of these eigenfunctions shows that they have a power law decay as s goes to infinity, and hence do not have moments of all orders. Hence our definition of the domain also excludes this continuous spectrum.

We now give a definition of a ladder operator of $\mathcal{D}$.

Definition 1. An operator $\mathcal{L}$ is a ladder operator for $\mathcal{D}$ with increment $\mu$ if $[\mathcal{D}, \mathcal{L}]=\mu \mathcal{L}$ for some $\mu \in \mathbb{C}$, where $[\cdot, \cdot]$ denotes the commutator $[\mathcal{D}, \mathcal{L}]=$ $\mathcal{D} \mathcal{L}-\mathcal{L} \mathcal{D}$.

The following lemma shows that ladder operators can be used to generate new eigenfunctions from ones that we already know.

Lemma 1. Suppose $\mathcal{L}$ is a ladder operator such that $[\mathcal{D}, \mathcal{L}]=\mu \mathcal{L}$. Let $\phi$ be an eigenfunction of $\mathcal{D}$ with eigenvalue $\chi$. Then either $\mathcal{L} \phi=0$, or $\mathcal{L} \phi$ is an eigenfunction of $\mathcal{D}$ with eigenvalue $\chi+\mu$.

Proof. We have $\mathcal{D} \mathcal{L} \phi-\mathcal{L} \mathcal{D} \phi=\mu \mathcal{L} \phi$. Since $\phi$ is an eigenfunction of $\mathcal{D}$, this gives us $\mathcal{D} \mathcal{L} \phi=(\chi+\mu) \mathcal{L} \phi$.

We defined the domain of $\mathcal{D}$ to be the set of functions that have moments of all orders. It should be noted that Lemma 1 would not apply if the domain had been (for example) the set of all square integrable functions. In that case a third possibility would exist. It could be that the function $\phi$ is square integrable, but the function $\mathcal{L} \phi$ is not. Thus $\mathcal{L} \phi$ would not generate a new eigenfunction.

We will show that $\mathcal{D}$ has $2 n+1$ ladder operators $\mathcal{L}_{ \pm k}, k=0, \ldots, n$. We begin by decomposing $\mathcal{D}$ into simple differential and multiplicative operators. 
Definition 2. We define the operators $L_{j}, j=1, \ldots, 2 n+1$ as follows.

$$
\begin{array}{ll}
L_{j} \phi=\partial_{s_{j}} \phi & \text { for } j=1, \ldots, n \\
L_{j+n} \phi=s_{j} \phi & \text { for } j=1, \ldots, n \\
L_{2 n+1} \phi=\mathcal{I} \phi & \text { for } j=2 n+1
\end{array}
$$

Here $\mathcal{I}$ is the identity operator. Note that $\left[L_{j}, L_{k}\right]=0$ unless $|j-k|=n$, and $\left[L_{j}, L_{j+n}\right]=\mathcal{I}$. In particular, we have

$$
\left[L_{j}, L_{k+n}\right]=\delta_{j k} \mathcal{I} \quad j, k=1, \ldots, n
$$

We note that $\mathcal{D}$ can be expressed in the operators $L_{j}$ as

$$
\mathcal{D}=\sum_{k, j=1}^{2 n+1} \frac{1}{2} d_{j k} L_{j} L_{k}
$$

We let $\mathbf{D}$ denote the symmetric matrix with components $d_{j k}$ in (21). The choice of $d_{j k}$ in (21) is not unique, but we make an explicit choice that makes this matrix symmetric. If we let $\mathbf{A}$ denote the antisymmetric matrix with components $a_{j k}$ given by

$$
\left[L_{j}, L_{k}\right]=a_{j k} \mathcal{I}
$$

then we have explicit expressions for $\mathbf{A}$ and $\mathbf{D}$

$$
\mathbf{A}=\left(\begin{array}{ccc}
\mathbf{0}_{n} & \mathbf{I}_{n} & 0 \\
-\mathbf{I}_{n} & \mathbf{0}_{n} & \vdots \\
0 & \cdots & 0
\end{array}\right), \quad \mathbf{D}=\left(\begin{array}{ccc}
\mathbf{B} & -\mathbf{H} & 0 \\
-\mathbf{H}^{T} & \mathbf{0}_{n} & \vdots \\
0 & \cdots & -\operatorname{tr}(\mathbf{H})
\end{array}\right)
$$

For details regarding the construction of $\mathbf{D}$ see Lemma 14 in Appendix A.

Just as $\mathcal{D}$ has a representation in terms of the operators $L_{j}$, its ladder operators will also be expressed in terms of the $L_{j}$. Consider an operator

$$
\mathcal{L}=\sum_{j=1}^{2 n+1} y_{j} L_{j}
$$

We write $\mathbf{y}$ for the vector of coefficients of $\mathcal{L}$. From the representations (21) and (24), we see that the commutator $[\mathcal{D}, \mathcal{L}]$ involves sums of terms of the form $L_{i} L_{j} L_{k}-L_{k} L_{i} L_{j}$, which do not at first sight appear to be linear in the operators $L_{m}, m=1, \ldots, 2 n+1$. However, by twice applying the commutator relations in equation (20) we can show that $L_{i} L_{j} L_{k}-L_{k} L_{i} L_{j}$ is in fact a sum of the $L_{m}$. Determining the coefficient vector $\mathbf{y}$ and increment $\mu$ thus becomes a matrix eigenvalue problem. The details of how we arrive at this form are given in Appendix A. Here we will merely state the result of these manipulations.

Lemma 2. If $\mathbf{y}$ is the vector of coefficients for $\mathcal{L}$, as defined as in equation (24), then the equation $[\mathcal{D}, \mathcal{L}]=\mu \mathcal{L}$ can be written as a matrix eigenvalue problem $\mathbf{T y}=\mu \mathbf{y}$, where $\mathbf{T}=\mathbf{D A}$, and $\mathbf{D}$ and $\mathbf{A}$ are defined in (23). 
We make the assumption that the eigenvalues of $\mathbf{H}$ have negative real parts, and the eigenvectors form a complete set. For simplicity of the arguments, we will also assume that the eigenvalues of $\mathbf{H}$ are simple. By explicitly writing out the eigenvalue problem $\mathbf{T y}=\mu \mathbf{y}$ we can determine the eigenvalues $\mu_{k}$ in terms of the eigenvalues of the matrix $\mathbf{H}$. We will give the details of the proof in Appendix A.

Lemma 3. The eigenvalues of $\mathbf{T}=\mathbf{D A}$ are $\left\{0, \pm \mu_{k}\right\}, k=1, \ldots, n$, where $-\mu_{k}$ are the eigenvalues of the matrix $\mathbf{H}$.

Note that $\mathcal{L}_{0}$ is the identity operator with increment 0 . Thus, our analysis only involves the $2 n$ ladder operators $\mathcal{L}_{ \pm k}$ for $k=1, \ldots, n$.

In doing the perturbation expansion it will be necessary to have the commutator relations of the operators $\mathcal{L}_{i}$. Finding the commutator relations for $\left[\mathcal{L}_{j}, \mathcal{L}_{k}\right]$ can be turned into a linear algebra problem involving the eigenvectors of the matrix T. In particular, using equations (24) and (22) we get

$$
\left[\mathcal{L}_{j}, \mathcal{L}_{k}\right]=\left(\mathbf{y}_{j}^{T} \mathbf{A y}_{k}\right) \mathcal{I}
$$

From equation (23) it is easily seen that

$$
\mathbf{A D}=-(\mathbf{D A})^{T}=-\mathbf{T}^{T}
$$

If $\mathbf{D A y}=\mu \mathbf{y}$, then multiplying both sides of this by $\mathbf{A}$ and using equation (26) we see that $\mathbf{A y}$ is an eigenvector of $\mathbf{T}^{T}$ with eigenvalue $-\mu$. With this in mind, the right hand side of equation (25) can be written as the inner product between the vector $\mathbf{y}_{j}$ and the adjoint eigenvector of $\mathbf{T}$ associated with $-\mu_{k}$. Using the fact that the eigenvectors and adjoint eigenvectors of a matrix form a bi-orthogonal set, we can arrive at a simple expression for the commutators.

When dealing with complex quantities, the notation in this argument gets to be a bit tedious, and we will leave the details to Appendix A. The final commutator result is given by the following lemma.

Lemma 4. For $j, k \geq 1$ we have $\left[\mathcal{L}_{j}, \mathcal{L}_{k}\right]=0$ and $\left[\mathcal{L}_{-j}, \mathcal{L}_{k}\right]=\delta_{j k} \mathcal{I}$.

In Dirac's theory of the harmonic oscillator, he shows that the Hamiltonian operator can be written as the product of the raising and lowering operators. We now generalize this result to the vector case. In this case the operator $\mathcal{D}$ can be written as a weighted sum of the products of the raising and lowering operators. The next lemma shows that the weights are in fact the eigenvalues $\mu_{k}$ of the matrix $\mathbf{T}$. We leave the proof of this lemma to Appendix A, but note that its proof is probably the most subtle one in this paper.

Lemma 5. The differential operator $\mathcal{D}=\sum_{i, j=1}^{2 n+1} \frac{1}{2} d_{i, j} L_{i} L_{j}$ can be written as

$$
\mathcal{D}=\sum_{k=1}^{n} \mu_{k} \mathcal{L}_{-k} \mathcal{L}_{k} .
$$

An important feature of the decomposition (27) is that only terms of the form $\mathcal{L}_{-k} \mathcal{L}_{k}, k>0$, appear (there are no terms of the form $\mathcal{L}_{k} \mathcal{L}_{-k}$ for $k>0$ ). 


\section{Eigenvalues and Eigenfunctions of $\mathcal{D}$}

In this section we will use the ladder operator formalism to completely characterize the eigenvalues and eigenfunctions of the operator $\mathcal{D}$. We note that the spectrum of $\mathcal{D}$ has already been studied and characterized [17, 18, 22, but not in terms of ladder operators. We include another proof of those results because the characterization in terms of ladder operators is used in the perturbation analysis in $\$ 4$

As with Dirac's theory of the quantum harmonic oscillator, the analysis of the spectrum using ladder operators requires that the real part of the spectrum is bounded above. We will now state this as a lemma, but leave the proof to Appendix B.

Lemma 6. The real part of spectrum of the operator $\mathcal{D}$, as defined in (15), is bounded above.

The following theorem will allow us to characterize the eigenfunction associated with the eigenvalue with the largest real part.

Theorem 1. Let $\Phi(\mathbf{s})$ be an eigenfunction of $\mathcal{D}$ (as in equation (15)) associated with the eigenvalue having the largest real part. We must have $\mathcal{L}_{k} \Phi=0$ for $k=1, \ldots, n$.

Proof. Suppose $\Phi(\mathbf{s})$ is an eigenfunction of $\mathcal{D}$ with eigenvalue $\chi$. If $\Psi=\mathcal{L}_{k} \Phi \neq$ 0 , then $\Psi(\mathbf{s})$ will be an eigenfunction of $\mathcal{D}$ with eigenvalue $\chi+\mu_{k}$. This will give us an eigenvalue with a larger real part than $\chi$. Hence if $\chi$ is the eigenvalue with the largest real part, then $\mathcal{L}_{k} \Phi=0$ for all $k$.

Remark 1 . The system $\mathcal{L}_{k} \Phi=0$ for each $k=1, \ldots, n$ is an over-determined system of first order differential equations. The fact that a solution exists is non-trivial. However, the fact that $\left[\mathcal{L}_{k}, \mathcal{L}_{j}\right]=0$ implies that the Frobenius Theorem applies (see [1, 6]), which guarantees the system is solvable.

Remark 2. As in the comment following Lemma 11. we should note that the domain of $\mathcal{D}$ is defined as the set of functions that have moments of all orders. If the domain of $\mathcal{D}$ were defined using the less stringent requirement that the eigenfunctions were square integrable, it would not be necessary that $\mathcal{L}_{k} \Phi=0$ for all $k$. This is because in this case $\mathcal{L}_{k} \Phi$ does not have to generate a new eigenfunction. It could instead produce a function that is not square integrable.

By Theorem 1 the "top" eigenfunction $\Phi_{0}(\mathbf{s})$ (i.e. the eigenfunction associated to the largest eigenvalue of $\mathcal{D}$ ) must satisfy the equations $\mathcal{L}_{k} \Phi_{0}=0$. If $\mathbf{y}^{k}$ is the eigenvector of $\mathbf{T}$ associated with the eigenvalue $\mu_{k}$, and if $\mu_{k} \neq 0$, then the last component of $\mathbf{y}^{k}$ vanishes (see the proof of Lemma 3 in Appendix A). That is, we can write

$$
\mathbf{y}^{k}=\left(\begin{array}{c}
\mathbf{p}_{k} \\
\mathbf{q}_{k} \\
0
\end{array}\right)
$$


Using equation (24), and the definition of the operators $L_{k}$ in (19), the equations $\mathcal{L}_{k} \Phi_{0}=0$ can thus be written as

$$
\mathbf{p}_{k} \cdot \nabla \Phi_{0}+\left(\mathbf{q}_{k} \cdot \mathbf{s}\right) \Phi_{0}=0 \quad k=1, \ldots, n
$$

If we make the ansatz that $\Phi_{0}(\mathbf{s})=\exp \left(-\frac{1}{2} \mathbf{s}^{T} \mathbf{\Sigma} \mathbf{s}\right)$, then equations (29) will be satisfied if and only if

$$
\mathbf{P}^{T} \boldsymbol{\Sigma}=\mathbf{Q}^{T}
$$

where

$$
\mathbf{P}=\left[\mathbf{p}_{1}, \mathbf{p}_{2}, \ldots, \mathbf{p}_{n}\right], \quad \mathbf{Q}=\left[\mathbf{q}_{1}, \mathbf{q}_{2}, \ldots, \mathbf{q}_{n}\right] .
$$

If $\mathbf{P}$ is invertible, this gives us $\boldsymbol{\Sigma}=\left(\mathbf{P}^{T}\right)^{-1} \mathbf{Q}^{T}$. It is not clear that $\mathbf{P}$ is invertible, or that $\boldsymbol{\Sigma}$ is symmetric. However, under certain weak assumptions on $\mathbf{H}$ and $\mathbf{B}$ (see Definition 3 and Lemma 7 below) this will be the case. If these assumptions hold, it is convenient to write $\boldsymbol{\Sigma}^{-1}=\mathbf{P Q}^{-1}$. We now define the notion of a controllable pair.

Definition 3. The matrices $\mathbf{H}$ and $\mathbf{B}$ will be said to form a controllable pair if there is no nontrivial vector $\mathbf{z}$ such that $\mathbf{z}^{T} \mathbf{H}^{k} \mathbf{B}=0$ for $k=0, \ldots, n-1$. This is equivalent to requiring $\operatorname{rank} \mathbf{C}=n$, where $\mathbf{C}$ is the $n \times n^{2}$ matrix $\mathbf{C}=\left[\mathbf{B}, \mathbf{H B}, \ldots, \mathbf{H}^{n-1} \mathbf{B}\right]$.

In Appendix B we prove the following lemma.

Lemma 7. Assuming all of the eigenvalues of $\mathbf{H}$ have real parts less than zero, the eigenvectors of $\mathbf{H}$ are complete, and that $\mathbf{B}$ is positive semidefinite, then $\boldsymbol{\Sigma}^{-1}=\mathbf{P Q}^{-1}$ is symmetric and positive semi-definite. If $\mathbf{H}$ and $\mathbf{B}$ also form a controllable pair, then the matrix $\boldsymbol{\Sigma}^{-1}$ is positive definite, and hence the matrices $\boldsymbol{\Sigma}=\mathbf{Q P}^{-1}$ and $\mathbf{P}$ are non-singular.

Requiring (H, B) to be a controllable pair eliminates some "degenerate" types of filters. For instance, if $\mathbf{H}=\operatorname{diag}\left(-\mu_{1},-\mu_{2}\right)$ and $\mathbf{B}=\operatorname{diag}(1,0)$ then $(\mathbf{H}, \mathbf{B})$ is not a controllable pair. In this case, $s_{1}(t)$ is a scalar OrnsteinUhlenbeck process, but $s_{2}(t)$ is deterministic, so $\mathbf{s}(t)$ is not a genuine twodimensional Ornstein-Uhlenbeck process, but rather it is a one-dimensional process with an appended deterministic component.

Definition 4. We will say that the $n \times n$ real matrices $\mathbf{H}$ and $\mathbf{B}$ satisfy the basic conditions if

(i) $\mathbf{B}$ is symmetric and positive semi-definite

(ii) $\mathbf{H}$ has simple eigenvalues $\left\{-\mu_{k}\right\}_{k=1}^{n}$ with $\operatorname{Re}\left[\mu_{k}\right]>0$ for $k=1, \ldots, n$

(iii) (H, B) form a controllable pair (Def. [3).

The requirement of simple eigenvalues for $\mathbf{H}$ is for convenience and could be replaced with the requirement of a complete set of eigenvectors. 
Lemma 8. Assuming $\mathbf{H}$ and $\mathbf{B}$ satisfy the basic conditions (Def. 4), the eigenvalue $\chi_{0}$ with the largest real part of $\mathcal{D}$ is simple, and the eigenfunction $\Phi_{0}(\mathbf{s})$ associated with it is given by

$$
\Phi_{0}(\mathbf{s})=\exp \left(-\frac{1}{2}\langle\mathbf{s}, \mathbf{\Sigma} \mathbf{s}\rangle\right),
$$

where $\boldsymbol{\Sigma}=\mathbf{Q P}^{-1}$. Moreover, $\chi_{0}=0$.

Proof. Without loss of generality we look for solutions of the form $\Phi_{0}(\mathbf{s})=$ $e^{\psi_{0}(\mathbf{s})}$. In order to satisfy equations (29) we must have

$$
\mathbf{p}_{k} \cdot \nabla \psi_{0}+\left(\mathbf{q}_{k} \cdot \mathbf{s}\right)=0
$$

A direct calculations shows that $\psi_{0}=-\frac{1}{2}\langle\mathbf{s}, \boldsymbol{\Sigma} \mathbf{s}\rangle$ satisfies this equation. If we have another solution to this equation, say $\psi_{1}$, then the difference $\psi_{0}-\psi_{1}$ between these solutions will satisfy $\mathbf{p}_{k} \cdot \nabla\left(\psi_{0}-\psi_{1}\right)=0$, for $k=1, \ldots, n$. The vectors $\mathbf{p}_{k}$ are complete (they are the eigenvectors of $\mathbf{H}^{T}$ ), which implies that $\psi_{0}-\psi_{1}$ is a constant. This in turn implies that the eigenfunctions associated with each of the solutions $\psi_{0}(\mathbf{s})$ are multiples of each other, hence $\chi_{0}$ is simple.

From Lemma 6 the real part of the the spectrum of $\mathcal{D}$ is bounded above. From Lemma 5 , we can write $\mathcal{D}=\sum_{k=1}^{n} \mu_{k} \mathcal{L}_{-k} \mathcal{L}_{k}$. Hence, when we apply $\mathcal{D}$ to the eigenfunction $\Phi_{0}(\mathbf{s})$ associated with $\chi_{0}$, we will get $\mathcal{D} \Phi_{0}=0$ because $\mathcal{L}_{k} \Phi_{0}=0, k=1, \ldots, n$, by Theorem 1 Hence $\chi_{0}=0$.

Theorem 2. Let $\mathbf{H}$ and $\mathbf{B}$ satisfy the basic conditions (Def. 布). $\chi$ is an eigenvalue of $\mathcal{D}$ if and only if it can be written as in equation (12), where $k_{j}$ are non-negative integers, and $-\mu_{j}$ are the eigenvalues of $\mathbf{H}$. The eigenfunction associated with $\chi_{\mathbf{k}}$ is given by $\Phi_{\mathbf{k}}(\mathbf{s})=\mathcal{L}_{-1}^{k_{1}} \mathcal{L}_{-2}^{k_{2}} \ldots \mathcal{L}_{-n}^{k_{n}} \Phi_{0}(\mathbf{s})$. where $\Phi_{0}(\mathbf{s})$ is defined as in equation (32).

Proof. From Lemmas 6 and 8 the real part of the the spectrum of $\mathcal{D}$ is bounded above by 0 , and $\chi=0$ is an eigenvalue of $\mathcal{D}$ that has the form (12). If $\chi$ is any other eigenvalue, and $\Phi$ is its eigenfunction, then there must be at least one value of $k$ such that $\mathcal{L}_{k} \Phi \neq 0$. If this new eigenvalue has the form given in equation (12), then the previous one will too. We can keep carrying out this process obtaining eigenvalues with larger real parts. This process must eventually end since the real part of the spectrum is bounded above. The only way it can end is when we arrive at the largest eigenvalue, which we have already seen, is zero. This implies equation (12).

The argument in the last paragraph shows that any eigenvalue of $\mathcal{D}$ must be of the form (12). To show that any number $\chi_{\mathbf{k}}$ of this form must be an eigenvalue of $\mathcal{D}$ we show that $\Phi_{\mathbf{k}}(\mathbf{s})=\mathcal{L}_{-1}^{k_{1}} \mathcal{L}_{-2}^{k_{2}} \ldots \mathcal{L}_{-n}^{k_{n}} \Phi_{0}(\mathbf{s})$ is the eigenfunction associated with $\chi_{\mathbf{k}}$. This follows from Lemma 19 in Appendix B.

\section{Perturbation Method}

The marginal-moment equation (14) is derived by multiplying (13) by a monomial $\mathbf{x}^{\alpha}$ for some multi-index $\alpha$, then integrating with respect to $\mathbf{x}$. If this is 
done for each multi-index of order $p$, we derive a set of equations for the $p$ th marginal moments. If we collect the $p$ th marginal moments into a vector $\mathbf{m}$, we arrive at (14). The matrices $\boldsymbol{\Gamma}_{0}, \boldsymbol{\Gamma}_{1}$ depend not only on $\mathbf{A}_{0}, \mathbf{A}_{1}$, but also on our mapping of the $p$ th marginal moments into $\mathbf{m}$. For this reason, we do not write the explicit form of $\boldsymbol{\Gamma}_{0}, \boldsymbol{\Gamma}_{1}$ in this section, but we do write them out for the example of second marginal moments for the Mathieu equation in $\$ 5$.

We let $\boldsymbol{\phi}_{j}$ denote the eigenvectors of $\boldsymbol{\Gamma}_{0}$ with eigenvalues $\nu_{j}$. We let $\boldsymbol{\psi}_{j}$ be the normalized adjoint eigenvectors, so that $\overline{\boldsymbol{\psi}}_{k}^{T} \boldsymbol{\phi}_{j}=\left\langle\boldsymbol{\psi}_{k}, \boldsymbol{\phi}_{j}\right\rangle=\delta_{k j}$. We may assume without loss of generality that the $\nu_{j}$ are ordered so that $\operatorname{Re}\left[\nu_{1}\right] \geq \operatorname{Re}\left[\nu_{j}\right]$ for all $j$.

We expand the unknowns as series in $\varepsilon$,

$$
\lambda=\lambda_{0}+\varepsilon \lambda_{1}+\varepsilon^{2} \lambda_{2}+\ldots, \quad \mathbf{m}(\mathbf{s})=\mathbf{m}_{0}(\mathbf{s})+\varepsilon \mathbf{m}_{1}(\mathbf{s})+\varepsilon^{2} \mathbf{m}_{2}(\mathbf{s})+\ldots,
$$

and solve for the terms of these series. If we substitute these expansions into (16), and collect the zeroth-order terms, we get

$$
\lambda_{0} \mathbf{m}_{0}=\mathcal{D} \mathbf{m}_{0}+\boldsymbol{\Gamma}_{0} \mathbf{m}_{0} .
$$

The eigenfunctions of $\mathcal{D}$ are scalar-valued, and the eigenvectors of $\boldsymbol{\Gamma}_{0}$ are constant vectors. Assuming that both the eigenfunctions of $\mathcal{D}$ and the eigenvectors of $\boldsymbol{\Gamma}_{0}$ are complete, then the most general solution $\mathbf{m}_{0}$ to (35) will be a product of an eigenfunction of $\mathcal{D}$ with an eigenvector of $\boldsymbol{\Gamma}_{0}$, and $\lambda_{0}$ will be the sum of the eigenvalues of $\mathcal{D}$ and $\boldsymbol{\Gamma}_{0}$. We are interested in the largest eigenvalue, so we take

$$
\mathbf{m}_{0}(\mathbf{s})=\Phi_{0}(\mathbf{s}) \phi_{1},
$$

and $\lambda_{0}=\nu_{1}$ because 0 is the largest eigenvalue of $\mathcal{D}$ and $\mathcal{D} \Phi_{0}=0$ (Lemma 8), and $\nu_{1}$ was selected to have the largest possible real part (note that the choice of $\nu_{1}$ need not be unique).

The form of the forcing in (5) allows us to represent $\langle\mathbf{a}, \mathbf{s}\rangle$ in terms of the ladder operators. In particular, the parametric forcing by the linear filter $\langle\mathbf{a}, \mathbf{s}(t)\rangle$ results in the presence of the first-order polynomial $\langle\mathbf{a}, \mathbf{s}\rangle$ in the Fokker-Planck equation, and thus to the term $\varepsilon\langle\mathbf{a}, \mathbf{s}\rangle \boldsymbol{\Gamma}_{1} \mathbf{m}$ in the moment equation (16). Since the ladder operators, $\mathcal{L}_{ \pm k}$, are linear combinations of first-order operators $\partial_{s_{j}}$ and monomials $s_{j}$, it is reasonable to try to write $\langle\mathbf{a}, \mathbf{s}\rangle$ as a linear combination of $\mathcal{L}_{ \pm k}$. The completeness of the eigenvectors of $\mathbf{H}$ allows us to do this, greatly simplifying our perturbation analysis.

Lemma 9. If the eigenvectors of $\mathbf{H}$ are complete, and $\alpha_{k}, \beta_{k}$ are defined as in equation (85) (Appendix D), then

$$
\langle\mathbf{a}, \mathbf{s}\rangle=\sum_{k=1}^{n}\left(\alpha_{k} \mathcal{L}_{k}+\beta_{k} \mathcal{L}_{-k}\right) .
$$

The proof of Lemma 9 is in Appendix D. Formula (37) ensures that the coefficients $\alpha_{k}$ and $\beta_{k}$ will appear in the coefficients of the perturbation expansions (34). We show in Appendix $\mathrm{C}$ that the extended power spectral density, 
$G(z)$, of $\langle\mathbf{a}, \mathbf{s}(t)\rangle$ can also be expressed in terms of $\alpha_{k}$ and $\beta_{k}$ (Theorem 6). This allows us to derive a simple formula for the order $\varepsilon^{2}$ coefficient of $\lambda(\varepsilon)$ in terms of $G(z)$ (Theorem 3 ).

Recall that there are $J=\left(\begin{array}{c}N+p-1 \\ p\end{array}\right)$ distinct $p$ th order monomials in $N$ variables, and that $\boldsymbol{\Gamma}_{0}$ and $\boldsymbol{\Gamma}_{1}$ are $J \times J$ matrices. We will assume that $\boldsymbol{\Gamma}_{0}$ has a complete set of eigenvectors, which is the case for the Mathieu equation, and occurs whenever the eigenvectors of $\mathbf{A}_{0}$ are complete. The following lemma gives solvability conditions that will be used repeatedly in our analysis.

Lemma 10. Let $\mathbf{H}$ and $\mathbf{B}$ satisfy the basic conditions (Def. 4). Suppose that $\boldsymbol{\Gamma}_{0}$ has a complete set of eigenvectors $\left\{\boldsymbol{\phi}_{j}\right\}_{j=1}^{J}$, with eigenvalues $\nu_{j}$, normalized adjoint eigenvectors $\left\{\boldsymbol{\psi}_{j}\right\}_{j=1}^{J}$, and that $\Phi(s)$ is an eigenfunction of $\mathcal{D}$ with eigenvalue $-\mu$. If $\mu \neq 0$, then then the equation

$$
\left(\lambda_{0}-\mathcal{D}-\boldsymbol{\Gamma}_{0}\right) \mathbf{m}=\Phi(\mathbf{s}) \mathbf{b}
$$

has a solution given by

$$
\mathbf{m}(\mathbf{s})=\Phi(\mathbf{s}) \sum_{j=1}^{J} \frac{\left\langle\boldsymbol{\psi}_{j}, \mathbf{b}\right\rangle}{\nu_{1}-\nu_{j}+\mu} \boldsymbol{\phi}_{j}
$$

On the other hand, if $\mu=0$ (and hence $\Phi(s)=\Phi_{0}(\mathbf{s})$ ) and $\nu_{1} \neq \nu_{j}$ for $j>1$, then the equation

$$
\left(\lambda_{0}-\mathcal{D}-\boldsymbol{\Gamma}_{0}\right) \mathbf{m}=\Phi_{0}(\mathbf{s}) \mathbf{b}
$$

has a solution if and only if $\left\langle\boldsymbol{\psi}_{1}, \mathbf{b}\right\rangle=0$. In this case, the solution is

$$
\mathbf{m}(\mathbf{s})=\kappa \Phi_{0}(s) \boldsymbol{\phi}_{1}+\Phi_{0}(\mathbf{s}) \sum_{j=2}^{J} \frac{\left\langle\boldsymbol{\psi}_{j}, \mathbf{b}\right\rangle}{\nu_{1}-\nu_{j}} \boldsymbol{\phi}_{j} .
$$

where $\kappa$ is an arbitrary constant.

The constant $\kappa$ can be used to choose a normalization for $\mathbf{m}$. We do not need to choose a specific normalization for $\mathbf{m}$, so we set $\kappa=0$ because it is convenient. One can check that if $\mathbf{A}_{0}$ has a complete set of eigenvectors then $\boldsymbol{\Gamma}_{0}$ will too.

Proof. If $\mu \neq 0$, then when we write $\mathbf{b}$ in the $\phi_{j}$ basis, and make the ansatz $\mathbf{m}(\mathbf{s})=\Phi(\mathbf{s}) \mathbf{c}$, where $\mathbf{c}$ is a constant vector, we arrive at the expression for $\mathbf{m}$ in equation (38). If $\mu=0$, and hence $\Phi(\mathbf{s})=\Phi_{0}(\mathbf{s})$, then we cannot solve this equation if $\mathbf{b}$ has any component in the direction of $\phi_{1}$. This gives the compatibility condition $\left\langle\boldsymbol{\psi}_{1}, \mathbf{b}\right\rangle=0$. Assuming this holds, the solution is given by equation (39).

We will now describe the outline of the perturbation analysis. In order to help us describe the perturbation analysis we will use the following definition. 
Definition 5. We say a function $\mathbf{f}(\mathbf{s})$ is in $\mathcal{V}_{k}$ if it can be written as the sum of eigenfunctions of $\mathcal{D}$ times constant vectors, where each of the eigenfunctions is the product of $k$ or fewer ladder operators $\mathcal{L}_{-j}, j=1, \ldots, n$ applied to the eigenfunction $\Phi_{0}(\mathbf{s})$.

The following lemma will be used in our perturbation analysis.

Lemma 11. If $h(\mathbf{s}) \in \mathcal{V}_{k}$, then $g(\mathbf{s})=\langle\mathbf{a}, \mathbf{s}\rangle h(\mathbf{s})$ is in $\mathcal{V}_{k+1}$.

Proof. This is almost a direct consequence of Lemmas 4 and 9 From Lemma 9 we know that $g(s)$ can be written as a sum of terms involving $\mathcal{L}_{-j} h(\mathbf{s})$ and $\mathcal{L}_{j} h(\mathbf{s})$ where $j>0$. By definition, each of the terms $\mathcal{L}_{-j} h(\mathbf{s})$ are in $\mathcal{V}_{k+1}$. On the other hand, the commutator relations $\left[\mathcal{L}_{j}, \mathcal{L}_{-k}\right]=-\delta_{j k} \mathcal{I}$ from Lemma 4 . and the fact that $\mathcal{L}_{j} \Phi_{0}(\mathbf{s})=0$ for $j>0$, can be used to show that $\mathcal{L}_{j} h(\mathbf{s})$ is in $\mathcal{V}_{k-1}$ That is, $\mathcal{L}_{j}$ has either canceled out a previous term $\mathcal{L}_{-j}$ applied to $\Phi_{0}$, or it commutes with all of the previous operators applied to $\Phi_{0}$, yielding the zero function because $\mathcal{L}_{j} \Phi_{0}=0$ for $j>0$.

The perturbation analysis proceeds as follows. We have a zeroth-order solution $\mathbf{m}_{0}=\phi_{1} \Phi_{0}$, which is clearly in $\mathcal{V}_{0}$. We will see by induction, that the function $\mathbf{m}_{k}(\mathbf{s})$ will be in $\mathcal{V}_{k}$.

The equation at each higher order will be of the form

$$
\left(\lambda_{0}-\mathcal{D}-\boldsymbol{\Gamma}_{0}\right) \mathbf{m}_{k}=-\lambda_{k} \mathbf{m}_{0}+\mathbf{r}_{k-1}(s)
$$

where $\mathbf{r}_{k-1}(s)$ is function that can be computed using the $\mathbf{m}_{j}$ and $\lambda_{j}$ for $j<k$. In particular, we have

$$
\mathbf{r}_{k-1}(\mathbf{s})=-\sum_{j=1}^{k-1} \lambda_{j} \mathbf{m}_{k-j}+\langle\mathbf{a}, \mathbf{s}\rangle \boldsymbol{\Gamma}_{1} \mathbf{m}_{k-1}
$$

Assuming that for $j<k$ the functions $\mathbf{m}_{j}(\mathbf{s})$ are in $\mathcal{V}_{j}$, then Lemma 11 ensures that the term $\mathbf{r}_{k-1}(\mathbf{s})$ will be in $\mathcal{V}_{k}$. We can write

$$
\mathbf{r}_{k-1}(\mathbf{s})=\Phi_{0}(\mathbf{s}) \mathbf{b}_{k-1}+\hat{\mathbf{r}}_{k-1}(\mathbf{s})
$$

where the term $\hat{\mathbf{r}}_{k-1}(\mathbf{s})$ can be written as a sum of eigenfunctions of $\mathcal{D}$ times constant vectors, where none of the eigenfunctions is $\Phi_{0}(\mathbf{s})$. With this in mind we use Lemma 10 to see that we will be able to solve equation (40) if and only if $\lambda_{k}\left\langle\boldsymbol{\psi}_{1}, \boldsymbol{\phi}_{1}\right\rangle=\left\langle\boldsymbol{\psi}_{1}, \mathbf{b}_{k-1}\right\rangle$, and hence

$$
\lambda_{k}=\left\langle\boldsymbol{\psi}_{1}, \mathbf{b}_{k-1}\right\rangle .
$$

Once we have chosen $\lambda_{k}$ in this way, we can solve for $\mathbf{m}_{k}$, and it will clearly be in $\mathcal{V}_{k}$, thus allowing us to continue the process to the next value of $k$ by induction.

Terms in $\mathbf{r}_{k-1}(\mathbf{s})$ proportional to $\Phi_{0}$ can only arise at even steps in the process (i.e. equations for $\lambda_{2 j}, \mathbf{m}_{2 j}$ ) because $\mathcal{L}_{k} \Phi_{k}=-\Phi_{0}$ (see equation 42). These terms proportional to $\Phi_{0}$ must satisfy the compatibility condition $\left\langle\boldsymbol{\psi}_{1}, \mathbf{b}\right\rangle=0$ as in Lemma 10 . 


\subsection{First Order}

To simplify notation, we make the following definition.

Definition 6. The functions $\Phi_{k}(\mathbf{s})$ are defined as

$$
\Phi_{k}(\mathbf{s})=\mathcal{L}_{-k} \Phi_{0}(\mathbf{s}), \quad k=1, \ldots, n,
$$

where $\Phi_{0}(\mathbf{s})$ is the eigenfunction of $\mathcal{D}$ associated with the eigenvalue with the largest real part. Note that, from Lemma 4 and Theorem 1. we have

$$
\mathcal{L}_{k} \Phi_{k}(\mathbf{s})=-\Phi_{0}(\mathbf{s}), \quad k=1, \ldots, n,
$$

because $\mathcal{L}_{k} \Phi_{k}=\mathcal{L}_{k} \mathcal{L}_{-k} \Phi_{0}=\left(\mathcal{L}_{-k} \mathcal{L}_{k}-1\right) \Phi_{0}=-\Phi_{0}$.

Substituting (34) into (16) and collecting terms of order $\varepsilon$, we get the equation for $\mathbf{m}_{1}$

$$
\left(\lambda_{0}-\mathcal{D}-\boldsymbol{\Gamma}_{0}\right) \mathbf{m}_{1}=-\lambda_{1} \mathbf{m}_{0}+\langle\mathbf{a}, \mathbf{s}\rangle \boldsymbol{\Gamma}_{1} \mathbf{m}_{0} .
$$

It is not hard to show that the eigenvalue $\lambda(\varepsilon)=\lambda_{0}+\varepsilon \lambda_{1}+\ldots$ must be an even function of $\varepsilon$. This is also intuitive because the sign of $\varepsilon$ plays no role in (5). Thus, it is no surprise that $\lambda_{1}=0$.

Lemma 12. If $\mathbf{H}$ and $\mathbf{B}$ satisfy the basic conditions (Def. 4), we have $\lambda_{1}=0$ and

$$
\mathbf{m}_{1}=\sum_{k=1}^{n} \Phi_{k}(\mathbf{s}) \mathbf{c}_{k}
$$

where $\Phi_{k}$ is defined in (41), and

$$
\mathbf{c}_{k}=\sum_{j=1}^{J} \frac{\beta_{k}\left\langle\boldsymbol{\psi}_{j}, \boldsymbol{\Gamma}_{1} \phi_{1}\right\rangle}{\nu_{1}-\nu_{j}+\mu_{k}} \boldsymbol{\phi}_{j}
$$

Proof. Using (37) and that $\mathcal{L}_{k} \Phi_{0}=0, \mathcal{L}_{-k} \Phi_{0}=\Phi_{k}$, we have

$$
\langle\mathbf{a}, \mathbf{s}\rangle \boldsymbol{\Gamma}_{1} \mathbf{m}_{0}=\sum_{k=1}^{n} \beta_{k} \boldsymbol{\Gamma}_{1} \phi_{1} \Phi_{k}(\mathbf{s})
$$

Thus, the right side of (43) is a finite sum of terms proportional to $\Phi_{0}, \Phi_{1}, \ldots, \Phi_{n}$, and each term can be treated separately. We now apply Lemma 10 to equation (43) using equation (46). The only term proportional to $\Phi_{0}$ is $-\lambda_{1} \mathbf{m}_{0}$. But according Lemma 10 this means $\left\langle\boldsymbol{\psi}_{1}, \lambda_{1} \boldsymbol{\phi}_{1}\right\rangle=0$. Hence, $\lambda_{1}=0$. The expression in (38) applied to the $\Phi_{k}$ terms for $k>0$ gives the expression in (44) .

\subsection{Second Order}

Substituting (34) into (16) and collecting terms of order $\varepsilon^{2}$, we get the equation for $\mathbf{m}_{2}$

$$
\left(\lambda_{0}-\mathcal{D}-\boldsymbol{\Gamma}_{0}\right) \mathbf{m}_{2}=-\lambda_{2} \mathbf{m}_{0}+\langle\mathbf{a}, \mathbf{s}\rangle \boldsymbol{\Gamma}_{1} \mathbf{m}_{1} .
$$

The situation here is similar to that for $\mathbf{m}_{1}$, except that the terms proportional to $\Phi_{0}(\mathbf{s})$ come from $\mathbf{m}_{0}$ as well as terms of the form $\mathcal{L}_{k} \Phi_{k}(\mathbf{s})=-\Phi_{0}(\mathbf{s})$. 
Lemma 13. If $\mathbf{H}$ and $\mathbf{B}$ satisfy the basic conditions (Def. 4), the compatibility condition for 47 implies

$$
\lambda_{2}=-\sum_{k=1}^{n}\left\langle\boldsymbol{\psi}_{1}, \alpha_{k} \boldsymbol{\Gamma}_{1} \mathbf{c}_{k}\right\rangle
$$

where $\mathbf{c}_{k}$ is defined as in 45 .

Proof. Lemma 12 shows that $\mathbf{m}_{1}=\sum_{k=1}^{n} \Phi_{k}(\mathbf{s}) \mathbf{c}_{k}$. This fact, and an application of the result in Lemma 9 implies that

$$
\langle\mathbf{a}, \mathbf{s}\rangle \boldsymbol{\Gamma}_{1} \mathbf{m}_{1}=\left(\sum_{l=1}^{n} \alpha_{l} \mathcal{L}_{l}+\beta_{l} \mathcal{L}_{-l}\right) \boldsymbol{\Gamma}_{1} \sum_{k=1}^{n} \Phi_{k}(\mathbf{s}) \mathbf{c}_{k}=-\Phi_{0}(\mathbf{s})\left(\sum_{k=1}^{n} \alpha_{k} \boldsymbol{\Gamma}_{1} \mathbf{c}_{k}\right)+\ldots
$$

where the term on the right is the only term proportional to $\Phi_{0}$. We used (42) to write $\mathcal{L}_{k} \Phi_{k}(\mathbf{s})=\mathcal{L}_{k} \mathcal{L}_{-k} \Phi_{0}(\mathbf{s})=-\Phi_{0}(\mathbf{s})$.

Using the form of $\mathbf{m}_{0}$ in (36), the compatibility condition from Lemma 10 implies $-\lambda_{2}\left\langle\boldsymbol{\psi}_{1}, \boldsymbol{\phi}_{1}\right\rangle=\left\langle\boldsymbol{\psi}_{1}, \sum_{k=1}^{n} \alpha_{k} \boldsymbol{\Gamma}_{1} \mathbf{c}_{k}\right\rangle$. Hence

$$
\lambda_{2}=-\left\langle\boldsymbol{\psi}_{1}, \sum_{k=1}^{n} \alpha_{k} \boldsymbol{\Gamma}_{1} \mathbf{c}_{k}\right\rangle=-\sum_{k=1}^{n}\left\langle\boldsymbol{\psi}_{1}, \alpha_{k} \boldsymbol{\Gamma}_{1} \mathbf{c}_{k}\right\rangle .
$$

Computing the expression for $\mathbf{m}_{2}$ is a simple exercise, but we do not write it here. Continuing this process for higher order terms is straightforward, though grows more tedious with each successive order.

Lemma 13 allows us to compute $\lambda_{2}$, but a nice feature of the second order term $\lambda_{2}$, is that it can be expressed by a simple formula involving the extended power spectral density $G$ of the process $\langle\mathbf{a}, \mathbf{s}(t)\rangle$ (see Appendix C). We prove the following theorem in Appendix D.

Theorem 3. If $\operatorname{Re}\left[\nu_{1}-\nu_{j}+\mu_{k}\right]>0$ for each $j=1, \ldots, J$ and $k=1, \ldots, n$,

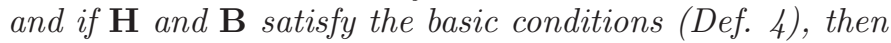

$$
\lambda_{2}=\sum_{j=1}^{J}\left\langle\boldsymbol{\psi}_{1}, \boldsymbol{\Gamma}_{1} \boldsymbol{\phi}_{j}\right\rangle\left\langle\boldsymbol{\psi}_{j}, \boldsymbol{\Gamma}_{1} \boldsymbol{\phi}_{1}\right\rangle G\left(\nu_{1}-\nu_{j}\right)
$$

Here $G(z)$ is the extended power spectral density of the forcing term $\langle\mathbf{a}, \mathbf{s}\rangle$.

Remark 3. Note that the coefficients $\left\langle\boldsymbol{\psi}_{1}, \boldsymbol{\Gamma}_{1} \boldsymbol{\phi}_{j}\right\rangle\left\langle\boldsymbol{\psi}_{j}, \boldsymbol{\Gamma}_{1} \boldsymbol{\phi}_{1}\right\rangle$ and the differences $\nu_{1}-\nu_{j}$ depend only on the differential equation for $\mathbf{x}$ (i.e. only on the matrices $\mathbf{A}_{0}$ and $\mathbf{A}_{1}$ ), and the function $G$ depends only on the filter $\langle\mathbf{a}, \mathbf{s}(t)\rangle$ (i.e. on $\mathbf{H}, \mathbf{B}, \mathbf{a}$ ). It would be interesting to investigate whether the same form as in (49) would hold for any asymptotically stationary filter. That is, if the expression for $\lambda_{2}$ would be a linear combination of values of $G$, where the coefficients depend only on the physical system, and the places where $G$ is evaluated are given by the eigenvalues of that system. 


\section{Applications}

\subsection{Second Moments for the Mathieu Equation}

We can write the Mathieu equation (1) as in (5) using a two-dimensional vector $\mathbf{x}^{T}=\left(x_{1}, x_{2}\right)$. In this case the matrices $\mathbf{A}_{0}, \mathbf{A}_{1}$ in equation (5) are

$$
\mathbf{A}_{0}=\left(\begin{array}{cc}
0 & 1 \\
-\omega_{0}^{2} & -\gamma
\end{array}\right), \quad \mathbf{A}_{1}=\left(\begin{array}{ll}
0 & 0 \\
1 & 0
\end{array}\right)
$$

We will consider the stability for the second moments. We define

$$
m_{j k}(\mathbf{s}, t)=\int_{\mathbb{R}^{2}} x_{j} x_{k} P\left(x_{1}, x_{2}, \mathbf{s}, t\right) d x_{1} d x_{2},
$$

In this case The Fokker Planck equation (13) can be written as

$$
\partial_{t} P=\mathcal{D} P-\frac{\partial}{\partial x_{1}}\left(x_{2} P\right)-\frac{\partial}{\partial x_{2}}\left(\left(-\omega_{0}^{2} x_{1}-\gamma x_{2}+\langle\mathbf{a}, \mathbf{s}\rangle x_{1}\right) P\right)
$$

If we multiply equation (52) by $x_{1}^{2}$, and integrate over all values of $x_{1}$ and $x_{2}$, after integrating by parts we get the equation

$$
\partial_{t} m_{11}=\mathcal{D} m_{11}+2 m_{12}
$$

Similarly multiplying equation (52) by $x_{1} x_{2}$ and $x_{2}^{2}$, integrating over all $x_{1}$ and $x_{2}$, and applying integration by parts, we get the equations

$$
\partial_{t} m_{12}=\mathcal{D} m_{12}-\omega_{0}^{2} m_{11}-\gamma m_{12}+m_{22}+\langle\mathbf{a}, \mathbf{s}\rangle m_{11}
$$

and

$$
\partial_{t} m_{22}=\mathcal{D} m_{22}-2 \omega_{0}^{2} m_{12}-2 \gamma m_{22}+2\langle\mathbf{a}, \mathbf{s}\rangle m_{12} .
$$

If we let $\mathbf{m}=\left(m_{11}, m_{12}, m_{22}\right)^{T}$ this can be written in the form of equation (14) where the matrices $\boldsymbol{\Gamma}_{0}, \boldsymbol{\Gamma}_{1}$ in (14) are given by

$$
\boldsymbol{\Gamma}_{0}=\left(\begin{array}{ccc}
0 & 2 & 0 \\
-\omega_{0}^{2} & -\gamma & 1 \\
0 & -2 \omega_{0}^{2} & -2 \gamma
\end{array}\right), \quad \boldsymbol{\Gamma}_{1}=\left(\begin{array}{ccc}
0 & 0 & 0 \\
1 & 0 & 0 \\
0 & 2 & 0
\end{array}\right)
$$

After assuming temporal behavior of the form $e^{\lambda t}$ we arrive at the eigenvalue problem

$$
\lambda \mathbf{m}=\mathcal{D} \mathbf{m}+\boldsymbol{\Gamma}_{0} \mathbf{m}+\varepsilon\langle\mathbf{a}, \mathbf{s}\rangle \boldsymbol{\Gamma}_{1} \mathbf{m} .
$$

for $\mathbf{m}(\mathbf{s})$ and $\lambda$, which is the same as (16). We will now apply the results of Theorem 3 to this set of equations.

In the case of second moments, the eigenvalues $\nu_{j}$ of $\boldsymbol{\Gamma}_{0}$ are given by sums of two eigenvalues of $\mathbf{A}_{0}$. I.e., $\nu_{j}=\sigma_{\ell}+\sigma_{m}$ where $\sigma_{k}$ are eigenvalues of $\mathbf{A}_{0}$. In the case of the Mathieu equation, the eigenvalues of $\mathbf{A}_{0}$ are $\sigma_{1}, \sigma_{2}$, where $\sigma_{1}=\bar{\sigma}_{2}=\frac{-\gamma+i \sqrt{4 \omega_{0}^{2}-\gamma^{2}}}{2}$. Hence, there are three choices of $\nu_{1}$, given by $\nu_{1}=-\gamma$, 
or $\nu_{1}=-\gamma \pm i \sqrt{4 \omega_{0}^{2}-\gamma^{2}}$, since they all have the same real part. In the case $\nu_{1}=-\gamma$, we have $\left\langle\boldsymbol{\psi}_{1}, \boldsymbol{\Gamma}_{1} \boldsymbol{\phi}_{1}\right\rangle=0$, so the $G(0)$ term does not appear. We also have $\left\langle\boldsymbol{\psi}_{1}, \boldsymbol{\Gamma}_{1} \boldsymbol{\phi}_{2}\right\rangle\left\langle\boldsymbol{\psi}_{2}, \boldsymbol{\Gamma}_{1} \boldsymbol{\phi}_{1}\right\rangle=\left\langle\boldsymbol{\psi}_{1}, \boldsymbol{\Gamma}_{1} \boldsymbol{\phi}_{3}\right\rangle\left\langle\boldsymbol{\psi}_{3}, \boldsymbol{\Gamma}_{1} \boldsymbol{\phi}_{1}\right\rangle=\frac{2}{4 \omega_{0}^{2}-\gamma^{2}}$. Hence

$$
\lambda_{2}=\frac{2}{4 \omega_{0}^{2}-\gamma^{2}}\left(G\left(\nu_{1}-\nu_{2}\right)+G\left(\nu_{1}-\nu_{3}\right)\right)=\frac{2}{4 \omega_{0}^{2}-\gamma^{2}} S\left(\sqrt{4 \omega_{0}^{2}-\gamma^{2}}\right)
$$

where $S(\omega)$ is the power spectral density of $\langle\mathbf{a}, \mathbf{s}(t)\rangle$. This follows because (without loss of generality, taking $\nu_{2}=-\gamma-i \sqrt{4 \omega_{0}^{2}-\gamma^{2}}=\bar{\nu}_{3}$ ) we have $\nu_{1}-\nu_{2}=$ $i \sqrt{4 \omega_{0}^{2}-\gamma^{2}}=-\left(\nu_{1}-\nu_{3}\right)$, and $G(i \omega)+G(-i \omega)=S(\omega)$ (see Appendix C).

If we take either $\nu_{1}=-\gamma \pm \sqrt{4 \omega_{0}^{2}-\gamma^{2}}$, then the expressions for $\lambda_{2}$ are

$$
\begin{aligned}
& (+) \quad \lambda_{2}=\frac{2}{4 \omega_{0}^{2}-\gamma^{2}}\left(G\left(i \sqrt{4 \omega_{0}^{2}-\gamma^{2}}\right)-2 G(0)\right) \\
& (-) \quad \lambda_{2}=\frac{2}{4 \omega_{0}^{2}-\gamma^{2}}\left(G\left(-i \sqrt{4 \omega_{0}^{2}-\gamma^{2}}\right)-2 G(0)\right) .
\end{aligned}
$$

Both cases have the same real part of $\lambda_{2}$

$$
\operatorname{Re}\left[\lambda_{2}\right]=\frac{1}{4 \omega_{0}^{2}-\gamma^{2}}\left(S\left(\sqrt{4 \omega_{0}^{2}-\gamma^{2}}\right)-2 S(0)\right)
$$

which is less than the expression in (55). Hence, we have proved

Theorem 4. If $\mathbf{H}$ and $\mathbf{B}$ satisfy the basic conditions (Def. 4), then the second moments of the Mathieu equation (1) become unstable when $\lambda(\varepsilon)>0$ where

$$
\lambda(\varepsilon)=-\gamma+\frac{2}{4 \omega_{0}^{2}-\gamma^{2}} S\left(\sqrt{4 \omega_{0}^{2}-\gamma^{2}}\right) \varepsilon^{2}+\ldots
$$

\subsection{Comparing Moments for the Mathieu Equation}

If we perform the same analysis as in $\$ 5.1$, but for the first and third marginal moment equations instead of the second marginal moment equation, we obtain results similar to Theorem 4. If we denote the largest eigenvalue of the $p$ th moment operator $\mathcal{D}+\boldsymbol{\Gamma}_{0}+\varepsilon\langle\mathbf{a}, \mathbf{s}\rangle \boldsymbol{\Gamma}_{1}$ as $\lambda^{(p)}$, then up to second order, we have

$$
\begin{aligned}
\lambda^{(1)}(\varepsilon)= & \frac{-\gamma+i \sqrt{4 \omega_{0}^{2}-\gamma^{2}}}{2}+\left(\frac{G\left(i \sqrt{4 \omega_{0}^{2}-\gamma^{2}}\right)}{4 \omega_{0}^{2}-\gamma^{2}}-\frac{G(0)}{4 \omega_{0}^{2}-\gamma^{2}}\right) \varepsilon^{2}+\ldots \\
\lambda^{(3)}(\varepsilon)= & \frac{-3 \gamma+i \sqrt{4 \omega_{0}^{2}-\gamma^{2}}}{2}+ \\
& \left(\frac{3 G\left(-i \sqrt{4 \omega_{0}^{2}-\gamma^{2}}\right)}{4 \omega_{0}^{2}-\gamma^{2}}+\frac{4 G\left(i \sqrt{4 \omega_{0}^{2}-\gamma^{2}}\right)}{4 \omega_{0}^{2}-\gamma^{2}}-\frac{G(0)}{4 \omega_{0}^{2}-\gamma^{2}}\right) \varepsilon^{2}+\ldots
\end{aligned}
$$


( $\boldsymbol{\Gamma}_{1}$ and $\boldsymbol{\Gamma}_{0}$ depend on $p$, but we do not make that explicit in our notation.) It is only the real parts of the eigenvalues that factor into the stability. We have

$$
\begin{aligned}
& \operatorname{Re}\left[\lambda^{(1)}(\varepsilon)\right]=-\frac{\gamma}{2}+\frac{1}{2\left(4 \omega_{0}^{2}-\gamma^{2}\right)}\left(S\left(\sqrt{4 \omega_{0}^{2}-\gamma^{2}}\right)-S(0)\right) \varepsilon^{2}+\ldots \\
& \operatorname{Re}\left[\lambda^{(2)}(\varepsilon)\right]=-\gamma+\frac{2}{4 \omega_{0}^{2}-\gamma^{2}} S\left(\sqrt{4 \omega_{0}^{2}-\gamma^{2}}\right) \varepsilon^{2}+\ldots \\
& \operatorname{Re}\left[\lambda^{(3)}(\varepsilon)\right]=-\frac{3}{2} \gamma+\frac{1}{2\left(4 \omega_{0}^{2}-\gamma^{2}\right)}\left(7 S\left(\sqrt{4 \omega_{0}^{2}-\gamma^{2}}\right)-S(0)\right) \varepsilon^{2}+\ldots
\end{aligned}
$$

In 24, there is a heuristic treatment of the first moments of $\mathbf{x}(t)$. There, Van Kampen writes a series for $\mathbf{x}(t)$, which he truncates at the $\varepsilon^{2}$ term and then averages to get an expression for $\langle\langle\mathbf{x}(t)\rangle\rangle$ up to order $\varepsilon^{2}$. He then points out that this new series is the solution to an ODE, up to order $\varepsilon^{2}$. The stability of $\langle\langle\mathbf{x}(t)\rangle\rangle$ is then analyzed in terms of this new ODE. His result for the Mathieu equation matches ours up to order $\varepsilon^{2}$ (although, he considers the case $\gamma=0$ ). Our result is a rigorous treatment, applies to higher moments, and we can find the solution to any order in $\varepsilon$. We stop at $\varepsilon^{2}$ in this paper only for convenience.

If we assume that the $\operatorname{Re}\left[\lambda^{(p)}\right]$ becomes positive while $\varepsilon$ is small (so we neglect the $\varepsilon^{4}$ terms and higher), then we can use (57), (58), and (59) to solve $\operatorname{Re}\left[\lambda^{(p)}\right]=0$ for $p=1,2,3$. Then we find that the second moments will become unstable before the first moments. If $S\left(\sqrt{4 \omega_{0}^{2}-\gamma^{2}}\right)>S(0)$, then the third moment will become unstable before the second moment. If $S\left(\sqrt{4 \omega_{0}^{2}-\gamma^{2}}\right) \leq$ $S(0)$, then the second moment becomes unstable before the third.

\subsection{Numerical Results}

In this subsection we discuss the computation of the eigenvalue that determines the stability of the Mathieu equation (1), with $\mathbf{A}_{0}, \mathbf{A}_{1}$ from (50) and $\boldsymbol{\Gamma}_{0}, \boldsymbol{\Gamma}_{1}$ from (53). We do not restrict ourselves to small values of $\varepsilon$. We carry out these calculations by converting the eigenvalue problem to an infinite dimensional system of linear equations, and truncating this system after a finite number of terms. Our procedure converges rapidly as the number of terms in our expansion is increased.

We limit ourselves to the case of a second-order filter given by (2), with $\mathbf{H}$, $\mathbf{B}$, and $\mathbf{a}$ given by

$$
\mathbf{H}=\left(\begin{array}{cc}
-\mu_{1} & 0 \\
\beta & -\mu_{2}
\end{array}\right), \quad \mathbf{B}=\left(\begin{array}{ll}
1 & 0 \\
0 & 0
\end{array}\right), \quad \mathbf{a}=\left(\begin{array}{l}
a_{1} \\
a_{2}
\end{array}\right),
$$

where $\beta, a_{1}, a_{2} \in \mathbb{R}, \beta \neq 0$, and $\mu_{1}, \mu_{2}>0$. The vector of second marginal moments $\mathbf{m}(\mathbf{s})$, given by (151), satisfies

$$
\begin{aligned}
\lambda \mathbf{m} & =\mathcal{D} \mathbf{m}+\boldsymbol{\Gamma}_{0} \mathbf{m}+\varepsilon\langle\mathbf{a}, \mathbf{s}\rangle \boldsymbol{\Gamma}_{1} \mathbf{m} \\
& =\frac{1}{2} \partial_{s_{1}}^{2} \mathbf{m}+\mu_{1} \partial_{s_{1}}\left(s_{1} \mathbf{m}\right)-\partial_{s_{2}}\left(\left(\beta s_{1}-\mu_{2} s_{2}\right) \mathbf{m}\right)+\boldsymbol{\Gamma}_{0} \mathbf{m}+\varepsilon\langle\mathbf{a}, \mathbf{s}\rangle \boldsymbol{\Gamma}_{1} \mathbf{m} .
\end{aligned}
$$


If we multiply (61) by $s_{2}^{j}$ and integrate with respect to $d s_{2}$, then we get

$$
\begin{aligned}
\lambda \mathbf{m}_{j}=\frac{1}{2} \partial_{s_{1}}^{2} \mathbf{m}_{j}+\mu_{1} \partial_{s_{1}}\left(s_{1} \mathbf{m}_{j}\right)+j \beta s_{1} \mathbf{m}_{j-1}+ & \\
& -j \mu_{2} \mathbf{m}_{j}+\boldsymbol{\Gamma}_{0} \mathbf{m}_{j}+\varepsilon a_{1} s_{1} \boldsymbol{\Gamma}_{1} \mathbf{m}_{j}+\varepsilon a_{2} \boldsymbol{\Gamma}_{1} \mathbf{m}_{j+1},
\end{aligned}
$$

where

$$
\mathbf{m}_{j}\left(s_{1}\right)=\int_{\mathbb{R}} s_{2}^{j} \mathbf{m}\left(s_{1}, s_{2}\right) d s_{2} .
$$

This is an infinite set of equations for the marginals $\left\{\mathbf{m}_{j}\left(s_{1}\right)\right\}$. Let $\varphi_{k}\left(s_{1}\right)=$ $H_{k}\left(\sqrt{\mu_{1}} s_{1}\right) e^{-\mu_{1} s_{1}^{2}}$, where $H_{k}$ is the $k$ th Hermite polynomial. We expand $\mathbf{m}_{j}$ in the basis $\varphi_{k}$ as

$$
\mathbf{m}_{j}\left(s_{1}\right)=\sum_{k} \mathbf{c}_{j}^{k} \varphi_{k}\left(s_{1}\right) .
$$

The $\varphi_{k}$ are eigenfunctions of the differential operator in the $s_{1}$ variable in (62); explicitly

$$
\frac{1}{2} \partial_{s_{1}}^{2} \varphi_{k}+\mu_{1} \partial_{s_{1}}\left(s_{1} \varphi_{k}\right)=-k \mu_{1} \varphi_{k} \quad k \geq 0 .
$$

The Hermite polynomials satisfy the recursion relation $H_{k+1}(y)=2 y H_{k}(y)-$ $2 k H_{k-1}(y)$, hence

$$
s_{1} \varphi_{k}\left(s_{1}\right)=\frac{1}{2 \sqrt{\mu_{1}}}\left(\varphi_{k+1}\left(s_{1}\right)+2 k \varphi_{k-1}\left(s_{1}\right)\right) \quad k \geq 0 .
$$

Thus, (62) simplifies and becomes an equation for $\mathbf{c}_{j}^{k}$

$$
\begin{aligned}
& \lambda \mathbf{c}_{j}^{k}=\left(\boldsymbol{\Gamma}_{0}-\left(k \mu_{1}+j \mu_{2}\right) \mathbf{I}\right) \mathbf{c}_{j}^{k}+\frac{j \beta}{2 \sqrt{\mu_{1}}}\left(\mathbf{c}_{j-1}^{k-1}+2(k+1) \mathbf{c}_{j-1}^{k+1}\right) \\
& +\frac{\varepsilon a_{1}}{2 \sqrt{\mu_{1}}} \boldsymbol{\Gamma}_{1}\left(\mathbf{c}_{j}^{k-1}+2(k+1) \mathbf{c}_{j}^{k+1}\right)+\varepsilon a_{2} \boldsymbol{\Gamma}_{1} \mathbf{c}_{j+1}^{k}
\end{aligned}
$$

If we consider a finite number of moments $\mathbf{m}_{j}$ for $j \leq N_{m}$, and truncate the expansion in $\varphi_{k}$ at $k \leq N_{h}$, then we get an approximation to the doubly infinite system (63). This can be written as a matrix equation

$$
\mathbf{L} \mathbf{z}=\lambda \mathbf{z}
$$

where $\mathbf{L}$ is an $\left(N_{m} N_{h} J\right) \times\left(N_{m} N_{h} J\right)$ matrix. This eigenvalue problem can be solved quickly on a computer.

Table 1 shows the computed value of $\lambda(\varepsilon)$ for second moments, which is the largest eigenvalue of $\mathbf{L}$ in (64). That is, $\lambda(\varepsilon)$ is the largest eigenvalue for the Mathieu equation with filter (60) (in this case the largest eigenvalue is real). $E_{2}$ is the error from a second-order perturbation expansion. That is, $E_{2}(\varepsilon)=\left|\lambda_{0}+\lambda_{2} \varepsilon^{2}-\lambda(\varepsilon)\right|$ with $\lambda_{0}=-\gamma$ and $\lambda_{2}$ is given in equation (55). $E_{4}$ is the fourth-order error, $E_{4}(\varepsilon)=\left|\lambda_{0}+\lambda_{2} \varepsilon^{2}+\lambda_{4} \varepsilon^{4}-\lambda(\varepsilon)\right|$, where $\lambda_{4}$ is computed by performing the perturbation analysis to order four (the formula for $\lambda_{4}$ is not presented here). The method converges rapidly; the values of $\lambda(\varepsilon)$ in the table were computed for $N_{m}=7$ and $N_{h}=5$. 


\begin{tabular}{|c|c|c|c|}
\hline & $\lambda(\varepsilon)$ & $E_{2}$ & $E_{4}$ \\
\hline$\varepsilon=0.01$ & $-9.89 \times 10^{-3}$ & $5.74 \times 10^{-8}$ & $2.20 \times 10^{-11}$ \\
\hline$\varepsilon=0.05$ & $-7.20 \times 10^{-3}$ & $3.62 \times 10^{-5}$ & $3.44 \times 10^{-7}$ \\
\hline$\varepsilon=0.10$ & $1.65 \times 10^{-3}$ & $5.96 \times 10^{-4}$ & $2.21 \times 10^{-5}$ \\
\hline
\end{tabular}

Table 1: Values of the error in computing $\lambda(\varepsilon)$ (for second moments) for three values of $\varepsilon . E_{2}$ is the error from the second order expansion, and $E_{4}$ is the error from the fourth-order expansion. Parameter values: $\mu_{1}=1.8, \mu_{2}=0.9, \beta=$ $1, \gamma=0.01, \omega_{0}=0.5, a_{1}=1, a_{2}=0.9, N_{m}=7, N_{h}=5$.

\subsection{Alternative Representation of $\lambda_{2}$}

We present a formula for $\lambda_{2}$ that involves only $\mathbf{A}_{0}$ and $\mathbf{A}_{1}$, avoiding construction of $\boldsymbol{\Gamma}_{0}, \boldsymbol{\Gamma}_{1}$. We do not present all of the details because the bookkeeping can be quite cumbersome (an interested reader can find the details in [8]), but we believe the formula for $\lambda_{2}$ will be useful for applications. For instance, if one wants to compute the perturbation coefficients on a computer, it is easy to build an algorithm based on equation (65) below, since one only needs to input the filter $(\mathbf{H}, \mathbf{B}, \mathbf{a})$ and the matrices $\mathbf{A}_{0}$ and $\mathbf{A}_{1}$.

The equation for the second marginal moments can be written as

$$
\partial_{t} \mathbf{M}=\mathcal{D} \mathbf{M}+\mathbf{A}_{0} \mathbf{M}+\mathbf{M} \mathbf{A}_{0}^{T}+\varepsilon\langle\mathbf{a}, \mathbf{s}\rangle\left(\mathbf{A}_{1} \mathbf{M}+\mathbf{M} \mathbf{A}_{1}^{T}\right)
$$

where $\mathbf{M}$ is the $N \times N$ symmetric matrix with $\mathbf{M}_{i j}=\int_{\mathbb{R}^{N}} x_{i} x_{j} P(\mathbf{s}, \mathbf{x}, t) d \mathbf{x}$. In this case one can solve an eigenvalue problem for the stability where we have eigenvalues and eigenmatrices. Looking for solutions of the form $\widetilde{\mathbf{M}}(\mathbf{s}, t)=$ $e^{\lambda t} \mathbf{M}(\mathbf{s})$, yields the eigenvalue problem for $\mathbf{M}(\mathbf{s})$

$$
\lambda \mathbf{M}=\mathcal{D} \mathbf{M}+\mathbf{A}_{0} \mathbf{M}+\mathbf{M} \mathbf{A}_{0}^{T}+\varepsilon\langle\mathbf{a}, \mathbf{s}\rangle\left(\mathbf{A}_{1} \mathbf{M}+\mathbf{M} \mathbf{A}_{1}^{T}\right) .
$$

The marginal moment tensor $\mathbf{M}$ is symmetric $\left(M_{j k}=M_{k j}\right)$, so we will use a basis of symmetric tensors to express $\mathbf{M}$, and in turn reproduce the results of \$4. The basis that is simplest is given by the eigenmatrices $\mathbf{E}_{j k}$ (and adjoints by $\mathbf{F}_{j k}$ with inner product $\langle\mathbf{E}, \mathbf{F}\rangle=\operatorname{tr}\left(\mathbf{E}^{T} \mathbf{F}\right)$ )

$$
\mathbf{E}_{j k}=\frac{1}{2}\left(\mathbf{h}_{j} \mathbf{h}_{k}^{T}+\mathbf{h}_{k} \mathbf{h}_{j}^{T}\right), \quad \mathbf{F}_{j k}=\frac{1}{2}\left(\mathbf{g}_{j} \mathbf{g}_{k}^{T}+\mathbf{g}_{k} \mathbf{g}_{j}^{T}\right),
$$

where $\mathbf{h}_{j}$ are eigenvectors of $\mathbf{A}_{0}$ with eigenvalues $\sigma_{j}$, and $\mathbf{g}_{k}$ are the normalized adjoint eigenvectors of $\mathbf{A}_{0},\left\langle\mathbf{g}_{j}, \mathbf{h}_{k}\right\rangle=\delta_{j k}$. The eigenvalues of the $\mathbf{E}_{j k}$ are sums of the $\sigma_{i} ; \mathbf{A}_{0} \mathbf{E}_{j k}+\mathbf{E}_{j k} \mathbf{A}_{0}^{T}=\left(\sigma_{j}+\sigma_{k}\right) \mathbf{E}_{j k}$.

The analogous result to Lemma 10 is straightforward to show, and following the steps in $₫$ we arrive at the following result (note that the eigenvalues $\nu_{j}$ of $\boldsymbol{\Gamma}_{0}$ from $\$ 4$ and $\$ 5.2$ are sums $\left.\sigma_{\ell}+\sigma_{m}\right)$.

Theorem 5. Let $\mathbf{H}$ and $\mathbf{B}$ satisfy the basic conditions (Def. 4), and let $\left\{\mathbf{h}_{j}\right\}_{j=1}^{N}$ form a complete set. For $q, r$ fixed, if $\operatorname{Re}\left[\sigma_{q}+\sigma_{r}-\sigma_{j}-\sigma_{k}+\mu_{\ell}\right]>0$ for each 
$j, k=1, \ldots, N$ and $\ell=1, \ldots, n$, then the order-two coefficient in the expansion $\lambda=\lambda_{0}+\lambda_{2} \varepsilon^{2}+\ldots$, with $\lambda_{0}=\sigma_{p}+\sigma_{r}$, is given by

$$
\lambda_{2}=8 \sum_{j, k=1}^{N} \frac{C_{j k q r} C_{q r j k}}{1+\delta_{q r}} G\left(\sigma_{q}+\sigma_{r}-\sigma_{j}-\sigma_{k}\right),
$$

where

$C_{j k \ell m}=\frac{1}{4}\left(\delta_{j m}\left\langle\mathbf{g}_{k}, \mathbf{A}_{1} \mathbf{h}_{\ell}\right\rangle+\delta_{k m}\left\langle\mathbf{g}_{j}, \mathbf{A}_{1} \mathbf{h}_{\ell}\right\rangle+\delta_{j \ell}\left\langle\mathbf{g}_{k}, \mathbf{A}_{1} \mathbf{h}_{m}\right\rangle+\delta_{k \ell}\left\langle\mathbf{g}_{j}, \mathbf{A}_{1} \mathbf{h}_{m}\right\rangle\right)$,

and $\mathbf{h}_{j}$ are eigenvectors of $\mathbf{A}_{0}$ with eigenvalues $\sigma_{j}$, and $\mathbf{g}_{k}$ are the normalized adjoint eigenvectors of $\mathbf{A}_{0},\left\langle\mathbf{g}_{j}, \mathbf{h}_{k}\right\rangle=\delta_{j k}$.

\section{Conclusions}

We have carried out a perturbation analysis to characterize the moment stability of parametrically forced linear equations, where the forcing is colored noise coming out of an Ornstein-Uhlenbeck process. Our analysis applies to arbitrary linear systems, and can in principle be carried out to any order. Our analysis depends on characterizing the spectrum of the vector Ornstein-Uhlenbeck process using ladder operators. Though this spectrum has been characterized elsewhere [17, 18, 22, we believe the ladder operator approach has been shown to be useful in carrying out our perturbation analysis.

\section{Acknowledgements}

We would like to thank John Torczynski for motivating and finding funding for this work. We also thank Jim Ellison, Nawaf Bou Rabee, and Rich Field for several fruitful discussions concerning stochastic differential equations.

\section{Appendix A: Supplementary Material for $\S 2$}

In this appendix we give several lemmas used in $\$ 2$ as well as supplying the proofs of several of the lemmas used in that section.

Lemma 14. The operator $\mathcal{D}$ defined in equation (15D) can be expressed as in equation (21), where the $d_{j k}$ are the components of the symmetric matrix $\mathbf{D}$, given in equation (23).

Proof. With $d_{j k}$ as the components of $\mathbf{D}$ given in equation (23), we have

$$
\frac{1}{2} \sum_{i=1}^{2 n+1} \sum_{j=1}^{2 n+1} d_{i j} L_{i} L_{j}=\frac{1}{2} \sum_{i=1}^{n} \sum_{j=1}^{n}\left(b_{i j} L_{i} L_{j}-h_{i j} L_{i} L_{j+n}-h_{j i} L_{i+n} L_{j}\right)-\frac{1}{2} \operatorname{tr}(\mathbf{H})
$$


The part of the operator involving the coefficients $b_{i j}$ is clearly equal to the operator $\frac{1}{2} \operatorname{div}(\mathbf{B} \nabla \cdot)$. To show that the left hand side of equation (66) is actually $\mathcal{D}$, we need to shows that the terms involving $h_{i j}$ are in fact the same as $-\sum_{i=1}^{n} \sum_{j=1}^{n} h_{i j} L_{i} L_{j+n}=-\operatorname{div}(\mathbf{H} \mathbf{s} \cdot)$. We compute

$$
\begin{aligned}
& \frac{1}{2} \sum_{i=1}^{n} \sum_{j=1}^{n}\left(h_{i j} L_{i} L_{j+n}+h_{j i} L_{i+n} L_{j}\right)+\frac{1}{2} \operatorname{tr}(\mathbf{H}) \\
& =\frac{1}{2} \sum_{i=1}^{n} \sum_{j=1}^{n}\left(h_{i j} L_{i} L_{j+n}+h_{i j} L_{j+n} L_{i}\right)+\frac{1}{2} \operatorname{tr}(\mathbf{H}) \\
& =\frac{1}{2} \sum_{i=1}^{n} \sum_{j=1}^{n}\left(h_{i j} L_{i} L_{j+n}+h_{i j}\left(L_{i} L_{j+n}-\delta_{i j}\right)\right)+\frac{1}{2} \operatorname{tr}(\mathbf{H})=\sum_{i=1}^{n} \sum_{j=1}^{n} h_{i j} L_{i} L_{j+n}
\end{aligned}
$$

In the second to last line above, we used the commutator relation from (20).

\section{Proof of Lemma 2}

Proof. We compute an expression for $[\mathcal{D}, \mathcal{L}]$ in terms of $\mathbf{D}$ and $\mathbf{A}$.

$$
\begin{aligned}
{[\mathcal{D}, \mathcal{L}] } & =\sum_{i, j, m} \frac{1}{2} d_{i j} y_{m}\left(L_{i} L_{j} L_{m}-L_{m} L_{i} L_{j}\right) \\
& =\sum_{i, j, m} \frac{1}{2} d_{i j} y_{m}\left(L_{i}\left[L_{j}, L_{m}\right]+\left[L_{i}, L_{m}\right] L_{j}\right) \\
& =\sum_{i, j, m} \frac{1}{2} d_{i j} y_{m}\left(L_{i} a_{j, m}+a_{i, m} L_{j}\right)=\sum_{i, m}\left(\frac{1}{2}\left(\mathbf{D}+\mathbf{D}^{T}\right) \mathbf{A}\right)_{i, m} y_{m} L_{i}
\end{aligned}
$$

For the equation $[\mathcal{D}, \mathcal{L}]=\mu \mathcal{L}$, this implies that we have

$$
\sum_{i, m}\left(\frac{1}{2}\left(\mathbf{D}+\mathbf{D}^{T}\right) \mathbf{A}\right)_{i, m} y_{m} L_{i}=\mu \sum_{i} y_{i} L_{i} .
$$

In matrix notation, this is just $\mathbf{D A y}=\mu \mathbf{y}$, because $\mathbf{D}=\mathbf{D}^{T}$.

This proof holds even if we do not assume that $\mathbf{D}$ is symmetric. In that case the analysis that follows would be done in terms of the symmetric matrix $\mathbf{S}=\frac{1}{2}\left(\mathbf{D}+\mathbf{D}^{T}\right)$, instead of $\mathbf{D}$. Thus, it is only for convenience that we use the symmetric form of $\mathbf{D}$ in (23).

\section{Proof of Lemma 3}

Proof. We denote the eigenvalues of $\mathbf{H}$ as $-\mu_{k}$ with $\operatorname{Re}\left[\mu_{k}\right]>0$ for $k=$ $1,2, \ldots, n$. Let $\mathbf{u}_{k}$ be the eigenvectors of $\mathbf{H}$ and $\mathbf{v}_{k}$ be the adjoint eigenvectors

$$
\mathbf{H u}_{k}=-\mu_{k} \mathbf{u}_{k}, \quad \mathbf{H}^{T} \mathbf{v}_{k}=-\bar{\mu}_{k} \mathbf{v}_{k}
$$


normalized so that

$$
\left\langle\mathbf{v}_{k}, \mathbf{u}_{j}\right\rangle=\delta_{j k}
$$

Recall that $\mathbf{H}$ is a real matrix, so complex eigenvalues come in complex conjugate pairs. If we write $\mathbf{y}=(\mathbf{p}, \mathbf{q}, r)^{T}$ then $\mathbf{T y}=\mu \mathbf{y}$ becomes

$$
\left(\begin{array}{ccc}
\mathbf{H} & \mathbf{B} & 0 \\
\mathbf{0}_{n} & -\mathbf{H}^{T} & 0 \\
0 & 0 & 0
\end{array}\right)\left(\begin{array}{l}
\mathbf{p} \\
\mathbf{q} \\
r
\end{array}\right)=\mu\left(\begin{array}{l}
\mathbf{p} \\
\mathbf{q} \\
r
\end{array}\right) .
$$

There is a solution with $\mu=0$ and $\mathbf{y}^{0}=(0, \ldots, 0,1)^{T}$. If $\mu \neq 0$ then $r=0$, and we have two cases. If $\mathbf{q}=\mathbf{0}$ then (68) reduces to $\mathbf{H p}=\mu \mathbf{p}$. Hence, $\mu=-\mu_{k}$ and $\mathbf{p}=\mathbf{u}_{k}$ for some $k$. We will denote this solution as $\mathbf{y}^{-k}=\left(\mathbf{u}_{k}, \mathbf{0}, 0\right)^{T}$. If $\mathbf{q} \neq \mathbf{0}$ then we must have $\mathbf{H}^{T} \mathbf{q}=-\mu \mathbf{q}$, so $\mu=\mu_{k}$ and $\mathbf{q}=\overline{\mathbf{v}}_{k}$ for some $k$. We denote the solution in this case as $\mathbf{y}^{k}=\left(-\left(\mathbf{H}-\mu_{k} \mathbf{I}\right)^{-1} \mathbf{B} \overline{\mathbf{v}}_{k}, \overline{\mathbf{v}}_{k}, 0\right)^{T}$.

Remark 4. T has the eigenvalue 0 , with corresponding ladder operator $\mathcal{L}_{0}=1$. This implies that $\mu_{0}=0$. However, in this degenerate case, it is convenient for notational purposes to define $\mu_{0}=-\operatorname{tr}(\mathbf{H})$. We will also write $\mu_{-k}$ in place of $-\mu_{k}$ to accommodate negative indices in the proof of Lemma 5 .

\section{The Proof of Lemma 4}

We denote by $\mathbf{w}^{ \pm k}$ the normalized adjoint eigenvectors of $\mathbf{T}$. That is, $\mathbf{T}^{T} \mathbf{w}^{ \pm k}= \pm \bar{\mu}_{k} \mathbf{w}^{ \pm k},\left\langle\mathbf{w}^{ \pm k}, \mathbf{y}^{ \pm k}\right\rangle=\delta_{j k}$, and $\left\langle\mathbf{w}^{ \pm k}, \mathbf{y}^{\mp k}\right\rangle=0$. We begin with a preliminary lemma.

Lemma 15. Let $\mathbf{u}_{k}$ and $\mathbf{v}_{k}$ be the eigenvectors of $\mathbf{H}$ as in equations $\sqrt{67}$. Let $\mathbf{y}^{ \pm k}, k=1, \ldots, n$, be the eigenvectors of $\mathbf{T}$ associated with the eigenvalue $\pm \mu_{k}$, and let $\mathbf{w}^{ \pm k}, k=1, \ldots, n$ be the normalized adjoint eigenvectors. Then for each $k=1, \ldots, n, \mathbf{A y}^{ \pm k}=\overline{\mathbf{w}}^{\mp k}$. For $k=0,1, \ldots, n, \mathbf{D w}^{ \pm k}=\bar{\mu}_{k} \overline{\mathbf{y}}^{\mp k}$ (using $\mu_{0}=-\operatorname{tr}(\mathbf{H})$ from Remark 4). Finally, $\sum_{k=-n}^{n} \bar{w}_{i}^{k} y_{j}^{k}=\delta_{i j}$.

Proof. Note that $\mathbf{y}^{ \pm k}$ are given explicitly in the proof of Lemma 3 and for $k \neq 0$

$$
\mathbf{y}^{-k}=\left(\mathbf{u}_{k}, \mathbf{0}, 0\right)^{T}, \quad \mathbf{y}^{k}=\left(-\left(\mathbf{H}-\mu_{k} \mathbf{I}\right)^{-1} \mathbf{B} \overline{\mathbf{v}}_{k}, \overline{\mathbf{v}}_{k}, 0\right)^{T} .
$$

We define

$$
\mathbf{w}^{k}=\left(\mathbf{0},-\overline{\mathbf{u}}_{k}, 0\right)^{T}, \quad \mathbf{w}^{-k}=\left(\mathbf{v}_{k},\left(\mathbf{H}-\bar{\mu}_{k} \mathbf{I}\right)^{-1} \mathbf{B v}_{k}, 0\right)^{T}
$$

and $\mathbf{y}^{0}=\mathbf{w}^{0}=(0, \ldots, 0,1)^{T}$. It is straightforward to check that $\left\langle\mathbf{w}^{ \pm j}, \mathbf{y}^{ \pm k}\right\rangle=$ $\delta_{j k},\left\langle\mathbf{w}^{ \pm j}, \mathbf{y}^{\mp k}\right\rangle=0, \mathbf{T}^{T} \mathbf{w}^{0}=\mathbf{0}$, and for $k \neq 0, \mathbf{T}^{T} \mathbf{w}^{ \pm k}= \pm \bar{\mu}_{k} \mathbf{w}^{ \pm k}$, so $\mathbf{w}^{ \pm k}$ are the normalized adjoint eigenvectors. Applying $\mathbf{A}$ to the $\mathbf{y}^{ \pm k}$ in (69) gives $\mathbf{A y}{ }^{ \pm k}=\overline{\mathbf{w}}^{\mp k}$ for $k \neq 0$, and hence applying $\mathbf{D}$ to $\mathbf{A} \mathbf{y}^{ \pm k}=\overline{\mathbf{w}}^{\mp k}$ gives $\mathbf{D w}^{ \pm k}=\bar{\mu}_{k} \overline{\mathbf{y}}^{\mp k}$ for $k \neq 0$. With $\mu_{0}=-\operatorname{tr}(\mathbf{H})=\bar{\mu}_{0}$ (since $\mathbf{H}$ is real), we have $\mathbf{D w}^{0}=\bar{\mu}_{0} \overline{\mathbf{y}}^{0}$. (Note that $\mathbf{A y}^{0}=\mathbf{0}$, so without the convention in Remark 4 we would not have $\mathbf{D w}^{0}=\bar{\mu}_{0} \overline{\mathbf{y}}^{0}$.)

We define the $(2 n+1) \times(2 n+1)$ matrices $\mathbf{Y}=\left[\mathbf{y}^{-n}, \ldots, \mathbf{y}^{n}\right]$ and $\mathbf{W}=$ $\left[\mathbf{w}^{-n}, \ldots, \mathbf{w}^{n}\right]$, then $\mathbf{W}^{*} \mathbf{Y}=\mathbf{I}_{2 n+1}$ because $\left(\overline{\mathbf{w}}^{i}\right)^{T} \mathbf{y}^{j}=\delta_{i j}$ for $-n \leq i, j \leq$ $n$. But this means $\mathbf{Y} \mathbf{W}^{*}=\mathbf{I}_{2 n+1}$ as well, and the components of $\mathbf{Y} \mathbf{W}^{*}$ are $\left(\mathbf{Y} \mathbf{W}^{*}\right)_{i j}=\sum_{k=-n}^{n} \bar{w}_{i}^{k} y_{j}^{k}$. 
We now give the proof of Lemma 4 .

Proof of Lemma 4 Recall A was defined as having coefficients $a_{m p}=\left[L_{m}, L_{p}\right]$. Writing out $\left[\mathcal{L}_{ \pm j}, \mathcal{L}_{k}\right]$ in terms of the $L_{m}$ we have

$$
\begin{aligned}
{\left[\mathcal{L}_{ \pm j}, \mathcal{L}_{k}\right] } & =\sum_{m, p=1}^{2 n+1} y_{m}^{ \pm j} y_{p}^{k}\left[L_{m}, L_{p}\right]=\sum_{m, p=1}^{2 n+1} y_{m}^{ \pm j} y_{p}^{k} a_{m p} \\
& =\left(\mathbf{y}^{ \pm j}\right)^{T} \mathbf{A} \mathbf{y}^{k}=\left\langle\overline{\mathbf{y}}^{ \pm j}, \mathbf{A} \mathbf{y}^{k}\right\rangle .
\end{aligned}
$$

Using $\mathbf{A} \mathbf{y}^{ \pm k}=\overline{\mathbf{w}}^{\mp k}$ we have $\left\langle\overline{\mathbf{y}}^{ \pm j}, \mathbf{A} \mathbf{y}^{k}\right\rangle=\left\langle\overline{\mathbf{y}}^{ \pm j}, \overline{\mathbf{w}}^{-k}\right\rangle=\overline{\left\langle\mathbf{y}^{ \pm j}, \mathbf{w}^{-k}\right\rangle}$. Hence, $\left[\mathcal{L}_{j}, \mathcal{L}_{k}\right]=\overline{\left\langle\mathbf{y}^{+j}, \mathbf{w}^{-k}\right\rangle}=0$ and $\left[\mathcal{L}_{-j}, \mathcal{L}_{k}\right]=\overline{\left\langle\mathbf{y}^{-j}, \mathbf{w}^{-k}\right\rangle}=\delta_{j k}$.

\section{The Proof of Lemma 5}

Proof of Lemma 囵. We first consider $\frac{\mu_{k}}{2} \mathcal{L}_{-k} \mathcal{L}_{k}=\sum_{p, m=1}^{2 n+1} \frac{\mu_{k}}{2} y_{m}^{-k} y_{p}^{k} L_{m} L_{p}$, for each $k=-n, \ldots, n$, using the conventions in Remark 4 . For each $k, \mathbf{D} \overline{\mathbf{w}}^{k}=$ $\mu_{k} \mathbf{y}^{-k}$, which follows from Lemma 15. Hence, $y_{m}^{-k}=\frac{1}{\mu_{k}} \sum_{q=1}^{2 n+1} d_{m q} \bar{w}_{q}^{k}$, so if we replace the term $y_{m}^{-k}$ in the above expression for $\frac{\mu_{k}}{2} \mathcal{L}_{-k} \mathcal{L}_{k}$, and sum over $k$, we get

$$
\begin{aligned}
\sum_{k=-n}^{n} \frac{\mu_{k}}{2} \mathcal{L}_{-k} \mathcal{L}_{k} & =\sum_{k=-n}^{n} \sum_{p, m, q=1}^{2 n+1} \frac{\mu_{k}}{2} \frac{1}{\mu_{k}} d_{m q} \bar{w}_{q}^{k} y_{p}^{k} L_{m} L_{p} \\
& =\sum_{p, m, q=1}^{2 n+1} \frac{1}{2} d_{m q} L_{m} L_{p} \sum_{k=-n}^{n} \bar{w}_{q}^{k} y_{p}^{k} .
\end{aligned}
$$

From Lemma 15] $\sum_{k=-n}^{n} \bar{w}_{q}^{k} y_{p}^{k}=\delta_{q p}$, so

$$
\sum_{k=-n}^{n} \frac{\mu_{k}}{2} \mathcal{L}_{-k} \mathcal{L}_{k}=\sum_{p, m, q=1}^{2 n+1} \frac{1}{2} d_{m q} \delta_{q p} L_{m} L_{p}=\sum_{p, m=1}^{2 n+1} \frac{1}{2} d_{m p} L_{m} L_{p}=\mathcal{D}
$$

For each $k>0$, we can write $\frac{\mu_{k}}{2} \mathcal{L}_{k} \mathcal{L}_{-k}=\frac{\mu_{k}}{2}\left(\mathcal{L}_{-k} \mathcal{L}_{k}-1\right)$ by the result of Lemma 4. Combining this with (71) and using $\mu_{0}=-\operatorname{tr}(\mathbf{H})$ we can write $\mathcal{D}$ as

$$
\mathcal{D}=-\frac{1}{2} \operatorname{tr}(\mathbf{H})+\sum_{k=1}^{n}\left\{\frac{\mu_{k}}{2} \mathcal{L}_{-k} \mathcal{L}_{k}+\frac{\mu_{k}}{2}\left(\mathcal{L}_{-k} \mathcal{L}_{k}-1\right)\right\} .
$$

But the eigenvalues of $\mathbf{H}$ are $-\mu_{k}$, hence $\operatorname{tr}(\mathbf{H})=-\sum_{k=1}^{n} \mu_{k}$ and we have $\mathcal{D}=\sum_{k=1}^{n} \mu_{k} \mathcal{L}_{-k} \mathcal{L}_{k}$.

\section{Appendix B: Supplementary Material for $\S 3$}

\section{Proof of Lemma 6}


Proof. Suppose $\chi$ is an eigenvalue of $\mathcal{D}$ with eigenfunction $\phi, \int_{\mathbb{R}^{n}}|\phi|^{2} d \mathbf{s}=1$. If we multiply (11) by $\bar{\phi}$, use the definition of $\mathcal{D}$ in (15), integrate over all of space, and integrate the term involving $\mathbf{B}$ by parts, we get

$$
\begin{aligned}
\chi \int_{\mathbb{R}^{n}}|\phi|^{2} d \mathbf{s}=\int_{\mathbb{R}^{n}} \bar{\phi} \mathcal{D} \phi d \mathbf{s} & =\int_{\mathbb{R}^{n}} \bar{\phi} \frac{1}{2} \operatorname{div}(\mathbf{B} \nabla \phi)-\bar{\phi} \operatorname{div}(\mathbf{H s} \phi) d \mathbf{s} \\
& =-\int_{\mathbb{R}^{n}} \frac{1}{2}\langle\nabla \phi, \mathbf{B} \nabla \phi\rangle+\bar{\phi} \operatorname{div}(\mathbf{H} \mathbf{s} \phi) d \mathbf{s}
\end{aligned}
$$

The matrix $\mathbf{B}$ is positive semi-definite, so $\langle\nabla \phi, \mathbf{B} \nabla \phi\rangle \geq 0$, hence $\operatorname{Re}[\chi] \leq$ $\operatorname{Re}\left[-\int_{\mathbb{R}^{n}} \bar{\phi} \operatorname{div}(\mathbf{H s} \phi) d \mathbf{s}\right]$. But, because $\mathbf{H}$ is real,

$$
2 \operatorname{Re}\left[\int_{\mathbb{R}^{n}} \bar{\phi} \operatorname{div}(\mathbf{H} \mathbf{s} \phi) d \mathbf{s}\right]=\int_{\mathbb{R}^{n}} \bar{\phi} \operatorname{div}(\mathbf{H s} \phi)+\phi \operatorname{div}(\mathbf{H s} \bar{\phi}) d \mathbf{s} .
$$

If we integrate the first term on the right in this expression by parts, and expand the second term we get

$$
\begin{aligned}
2 \operatorname{Re}\left[\int_{\mathbb{R}^{n}} \bar{\phi} \operatorname{div}(\mathbf{H s} \phi) d \mathbf{s}\right] & =\int_{\mathbb{R}^{n}}-\nabla \bar{\phi} \cdot(\phi \mathbf{H s})+\phi(\bar{\phi} \operatorname{tr}(\mathbf{H})+(\mathbf{H s}) \cdot \nabla \bar{\phi}) d \mathbf{s} \\
& =\int_{\mathbb{R}^{n}}|\phi|^{2} \operatorname{tr}(\mathbf{H}) d \mathbf{s}=\operatorname{tr}(\mathbf{H}) .
\end{aligned}
$$

Hence, $\operatorname{Re}[\chi] \leq-\frac{1}{2} \operatorname{tr}(\mathbf{H})$.

\section{Proof of Lemma 7}

The proof of Lemma 7 follows almost immediately from a few preliminary lemmas.

Lemma 16. Suppose the eigenvectors $\mathbf{q}_{k}$ of $\mathbf{H}$ are complete and the adjoint eigenvectors $\mathbf{p}_{k}$ are normalized so $\left\langle\mathbf{p}_{j}, \mathbf{q}_{k}\right\rangle=\delta_{j k}$. Let $\mathbf{P}=\left[\mathbf{p}_{1}, \mathbf{p}_{2}, \ldots, \mathbf{p}_{n}\right]$, $\mathbf{Q}=\left[\mathbf{q}_{1}, \mathbf{q}_{2}, \ldots, \mathbf{q}_{n}\right]$. We have

$$
\mathbf{H} \boldsymbol{\Sigma}^{-1}+\boldsymbol{\Sigma}^{-1} \mathbf{H}^{T}=-\mathbf{B}
$$

where $\boldsymbol{\Sigma}^{-1}=\mathbf{P Q}^{-1}$.

Remark 5. Lemmas 17 and 7 show that, with the appropriate assumptions on $\mathbf{H}$ and $\mathbf{B}$, the matrix $\mathbf{P}$ is invertible and thus there exists a nonsingular matrix $\boldsymbol{\Sigma}=\mathbf{Q P}^{-1}$, so our use of the notation $\boldsymbol{\Sigma}^{-1}$ is appropriate.

Proof. According to equation (68) we have $\mathbf{H} \mathbf{p}_{k}+\mathbf{B} \mathbf{q}_{k}=\mu_{k} \mathbf{p}_{k}$, and $-\mathbf{H}^{T} \mathbf{q}=$ $\mu_{k} \mathbf{q}_{k}$. Writing this out in matrix form we get $\mathbf{H P}+\mathbf{B Q}=\mathbf{P M},-\mathbf{H}^{T} \mathbf{Q}=\mathbf{Q M}$. Here $\mathbf{M}$ is the diagonal matrix with $\mu_{k}$ on the $k$ th diagonal. Using the second of these equations to write $\mathbf{M}$ in terms of $\mathbf{Q}$ and $\mathbf{H}$, and assuming $\mathbf{Q}$ is invertible (the eigenvectors of $\mathbf{H}$ are complete) we get $\mathbf{M}=-\mathbf{Q}^{-1} \mathbf{H}^{T} \mathbf{Q}$. Substituting this into the first equation we get $\mathbf{H P}+\mathbf{B Q}=-\mathbf{P Q}^{-1} \mathbf{H}^{T} \mathbf{Q}$. If we multiply this by $\mathbf{Q}^{-1}$ on the right and rearrange, we get the result of the lemma. 
We will use the following result for controllable pairs, which follows immediately from Theorem 2 in [10].

Lemma 17. If $\mathbf{B}$ is positive semi-definite, and the eigenvalues of $\mathbf{H}$ all have real parts less than zero, then the solution to $\mathbf{H R}+\mathbf{R H}^{T}=-\mathbf{B}$ is symmetric and positive definite provided $(\mathbf{H}, \mathbf{B})$ form a controllable pair.

Lemma 7 follows almost immediately from the previous two lemmas.

\section{Some lemmas used in the proof of Theorem 2}

Lemma 18. For any integer $m \geq 0$, the operators $\mathcal{L}_{k}$ and $\mathcal{L}_{-k}$ satisfy

$$
\left[\mathcal{L}_{-k}^{m+1}, \mathcal{L}_{k}\right]=(m+1) \mathcal{L}_{-k}^{m}
$$

Proof. For $m=0$, this follows immediately from Lemma 4. We can now proceed by induction. In particular, if $\mathcal{L}_{-k}^{m} \mathcal{L}_{k}-\mathcal{L}_{k} \mathcal{L}_{-k}^{m}=m \mathcal{L}_{-k}^{m-1}$, then if we multiply both sides of this equation by $\mathcal{L}_{-k}$ and use $\mathcal{L}_{-k}^{m} \mathcal{L}_{k} \mathcal{L}_{-k}=\mathcal{L}_{-k}^{m}\left(-I+\mathcal{L}_{-k} \mathcal{L}_{k}\right)=$ $\mathcal{L}_{-k}^{m+1} \mathcal{L}_{k}-\mathcal{L}_{-k}^{m}$, we find that $\mathcal{L}_{-k}^{m+1} \mathcal{L}_{k}-\mathcal{L}_{k} \mathcal{L}_{-k}^{m+1}=(m+1) \mathcal{L}_{-k}^{m}$, which proves the lemma.

Lemma 19. Let $\mathbf{H}$ and $\mathbf{B}$ satisfy the basic conditions (Def. 母), and let $\mathbf{k}=$ $\left(k_{1}, k_{2}, . . k_{n}\right)$ be a vector of nonnegative integers. Let

$$
\Phi_{\mathbf{k}}(\mathbf{s})=\mathcal{L}_{-1}^{k_{1}} \mathcal{L}_{-2}^{k_{2}} \ldots \mathcal{L}_{-n}^{k_{n}} \Phi_{0}(\mathbf{s}),
$$

then $\Phi_{\mathbf{k}}(\mathbf{s})$ is nonzero, and has an eigenvalue of

$$
\chi_{\mathbf{k}}=-\sum_{j=1}^{n} k_{j} \mu_{j}
$$

Proof. We begin by showing that $\mathcal{L}_{-k}^{m} \Phi_{0}(\mathbf{s})$ is nonzero for all $m \geq 0$. This clearly holds for $m=0$ by Lemma 8 By induction we can see that if it is nonzero for $m-1$, then it is non-zero for $m$. This follows from the fact that $\left[\mathcal{L}_{-k}^{m}, \mathcal{L}_{k}\right]=m \mathcal{L}_{-k}^{m-1}$, and the fact that $\mathcal{L}_{k} \Phi_{0}=0$. Combining these two facts we get $-\mathcal{L}_{k} \mathcal{L}_{-k}^{m} \Phi_{0}(\mathbf{s})=m \mathcal{L}_{-k}^{m-1} \Phi_{0}(\mathbf{s})$. This shows that if $\mathcal{L}_{-k}^{m}$ vanished,then $\mathcal{L}_{-k}^{m-1}$ would also have to vanish. Since we are assuming this is not the case, it follows that $\mathcal{L}_{-k}^{m} \Phi_{0}(\mathbf{s})$ does not vanish, and hence by induction does not vanish for any $m \geq 0$.

To show that a general function $\Phi_{\mathbf{k}}(\mathbf{s})$ does not vanish, we can proceed by a different induction proof. In particular, since the operator $\mathcal{L}_{-1}$ commutes with both $\mathcal{L}_{-2}$ and $\mathcal{L}_{2}$ we see that for any operator $Z$ of the form $Z=\mathcal{L}_{-1}^{p}$ where $p$ is a non-negative integer, we have $\left[Z \mathcal{L}_{-2}^{m}, \mathcal{L}_{2}\right]=m Z \mathcal{L}_{-2}^{m-1}$. We can now use almost the identical argument as in the last paragraph to show that any function of the form $Z \mathcal{L}_{-2}^{m} \Phi_{0}$ will be non-zero. We can now carry out this process by induction to see that any function of the form $\Phi_{\mathbf{k}}(\mathbf{s})$ will be nonzero.

Once we know that $\Phi_{\mathbf{k}}(\mathbf{s})$ is nonzero, it is clear from the ladder operator formalism that its eigenvalue must have the form in (75). 
There is one subtle point we would like to discuss in our proof of Theorem 2. Our proof relies on the fact that if $\phi$ is an eigenfunction of $\mathcal{D}$, then either $\mathcal{L}_{k} \phi=0$, or $\mathcal{L}_{k} \phi$ gives a new eigenfunction whose eigenvalue has a smaller real part. This relies on the assumption that $\mathcal{L}_{k} \phi$ remains in the domain of our operator. The domain of our operator consists of functions that have moments of all orders. Clearly, if this is true of $\phi$, this will be true of $\mathcal{L}_{k} \phi$. However, we must also make sure that the function $\mathcal{L}_{k} \phi$ has sufficient numbers of derivatives to satisfy our differential equation. This is clearly true of the eigenfunctions we have found. That is, they clearly have infinitely many derivatives. However, we should consider the possibility that there are other eigenfunctions that we have not accounted for that are not infinitely differentiable. General theorems on elliptic operators rule out such eigenfunctions if $\mathbf{B}$ is positive definite. However, we have only required that $\mathbf{B}$ be positive semi-definite, and that $\mathbf{H}$ and $\mathbf{B}$ form a controllable pair. A heuristic argument that we have found all of the eigenfunctions in this less restrictive case is as follows. If we perturb the matrix $\mathbf{B}$ to make it positive definite, then we know we have all of the eigenfunctions. As our perturbation parameter goes to zero, there is nothing unusual happening to our spectrum (such as eigenvalues going off to infinity, or clustering about a point). Hence, if the eigenfunctions are complete for positive definite $\mathbf{B}$ they are clearly complete in the less restrictive case where $\mathbf{B}$ and $\mathbf{H}$ form a controllable pair.

\section{Appendix C}

In this appendix, we provide formulas for the asymptotic autocorrelation function of the process $\mathbf{s}(t)$ and the extended power spectral density (defined in (79)) for $\mathbf{s}(t)$ as well as for the filter $\langle\mathbf{a}, \mathbf{s}(t)\rangle$. In particular, the results of Theorem 6 and Corollary 17, are used to express $\lambda_{2}$ in Theorems 3, 4, and 5, and throughout 95 . Corollary 7 gives a practical formula for computing the power spectral densities of $\mathbf{s}(t)$ and $\langle\mathbf{a}, \mathbf{s}(t)\rangle$.

\subsection{The Asymptotic Autocorrelation Function}

We begin by proving a lemma concerning the autocorrelation function of $\mathbf{s}(t)$ as defined in equation (2). $\mathbf{s}(t)$ is not a stationary process, but as $t \rightarrow \infty$ it approaches a stationary process, which we refer to as asymptotically stationary.

Lemma 20. Suppose $\mathbf{H}$ and $\mathbf{B}$ satisfy the basic conditions (Def. 4), and let $\mathbf{s}(t)$ be the solution to equation (2) with zero initial conditions. As $t \rightarrow \infty$ the autocorrelation function $\mathbf{R}(\tau)=\left\langle\left\langle\mathbf{s}(t) \mathbf{s}^{T}(t+\tau)\right\rangle\right\rangle$ is given by

$$
\mathbf{R}(\tau)=\boldsymbol{\Sigma}^{-1} e^{\mathbf{H}^{T} \tau} \quad \text { for } \tau>0,
$$

and $\boldsymbol{\Sigma}^{-1}=\mathbf{P Q}^{-1}$ satisfies equation (72). 
Proof. We define

$$
\mathbf{K}(t)=e^{\mathbf{H} t} \mathbf{B} e^{\mathbf{H}^{T} t}, \quad \mathbf{K}_{0}=\lim _{t \rightarrow \infty} \int_{0}^{t} \mathbf{K}(t-\sigma) d \sigma .
$$

The solution to equation (2) (with zero initial conditions) is given by

$$
\mathbf{s}(t)=\int_{0}^{t} e^{\mathbf{H}(t-s)} \boldsymbol{\xi}(s) d s
$$

We can write

$$
\mathbf{s}(t) \mathbf{s}^{T}(t+\tau)=\int_{0}^{t} \int_{0}^{t+\tau} e^{\mathbf{H}(t-s)} \boldsymbol{\xi}(s) \boldsymbol{\xi}^{T}(r) e^{\mathbf{H}^{\mathbf{T}}(t+\tau-r)} d r d s .
$$

If we take the expected value of both sides of this equation, and use the fact that $\left\langle\left\langle\boldsymbol{\xi}(s) \boldsymbol{\xi}^{T}(r)\right\rangle\right\rangle=\mathbf{B} \delta(r-s)$, we arrive at the equation

$$
\left\langle\left\langle\mathbf{s}(t) \mathbf{s}^{T}(t+\tau)\right\rangle\right\rangle=\int_{0}^{t} e^{\mathbf{H}(t-\sigma)} \mathbf{B} e^{\mathbf{H}^{T}(t-\sigma)} e^{\mathbf{H}^{T} \tau} d \sigma=\int_{0}^{t} \mathbf{K}(t-\sigma) d \sigma e^{\mathbf{H}^{T} \tau} .
$$

When deriving equation (78) we have assumed that the variable $r$ is equal to the variable $s$ at some point when doing the integration. This will only be guaranteed if $\tau>0$, and hence this is only valid for $\tau>0$. The expression for $\tau<0$, is obtained by using the fact that the autocorrelation function must satisfy $\mathbf{R}(-\tau)=\mathbf{R}^{T}(\tau)$.

Assuming that all of the eigenvalues of $\mathbf{H}$ have negative real part, the process $\mathbf{s}(t)$ will become stationary as $t \rightarrow \infty$. We take the limit of equation (78) as $t \rightarrow \infty$ to get

$$
\mathbf{R}(\tau)=\mathbf{K}_{0} e^{\mathbf{H}^{T} \tau},
$$

where $\mathbf{K}_{0}$ is defined in equation (77). We now show $\mathbf{K}_{0}=\boldsymbol{\Sigma}^{-1}$ by showing $\mathbf{K}_{0}$ satisfies equation (72), i.e. $\mathbf{H K}_{0}+\mathbf{K}_{0} \mathbf{H}^{T}=-\mathbf{B}$.

We have from (77)

$$
\frac{d}{d s} \mathbf{K}(s)=\mathbf{H K}(s)+\mathbf{K}(s) \mathbf{H}^{T} .
$$

It follows that

$$
\mathbf{H K}_{0}+\mathbf{K}_{0} \mathbf{H}^{T}=-\lim _{t \rightarrow \infty} \int_{0}^{t} \frac{d}{d s}(\mathbf{K}(t-s)) d s .
$$

We can evaluate this integral using the fundamental theorem of calculus. When we do this we find that the contribution at $s=0$ vanishes in the limit as $t \rightarrow \infty$. Since $\mathbf{K}(0)=\mathbf{B}$, the contribution at $s=t$ is just $-\mathbf{B}$, which completes the proof of the lemma. 


\subsection{The Extended Power Spectral Density}

The expression for the eigenvalue (with largest real part) of the perturbed operator $\mathcal{D}+\boldsymbol{\Gamma}_{0}+\varepsilon\langle\mathbf{a}, \mathbf{s}\rangle \boldsymbol{\Gamma}_{1}$ will be written in terms of the Laplace transform of the asymptotic autocorrelation function of the asymptotically stationary filter $\langle\mathbf{a}, \mathbf{s}(t)\rangle$, which we denote by $G$. $G$ can be viewed as an extension of the power spectral density, and has the advantage that it can be evaluated at points in the complex plane, outside of the domain of the power spectral density.

Definition 7. Let $\mathbf{s}(t)$ be an asymptotically stationary stochastic process (i.e. stationary in the limit $t \rightarrow \infty$ ) with asymptotic autocorrelation function $\mathbf{R}(\tau)$. We define the extended power spectral density of $\mathbf{s}(t)$ as

$$
\mathbf{G}(z)=\int_{0}^{\infty} \mathbf{R}(\tau) e^{-z \tau} d \tau .
$$

With this definition, the scalar filter $\langle\mathbf{a}, \mathbf{s}(t)\rangle$ has extended power spectral density $G(z)=\langle\mathbf{a}, \mathbf{G}(z) \mathbf{a}\rangle$. $\mathbf{G}$ is indeed an extension of the power spectral density $\mathbf{S}(\omega)=\int_{\mathbb{R}} \mathbf{R}(\tau) e^{-i \omega \tau} d \tau$, because the domain of $\mathbf{G}$ contains the set $\{z \in \mathbb{C}$ : $\operatorname{Re}[z] \geq 0\}$. In particular, $\operatorname{Re}[\mathbf{G}(i \omega)]=\frac{1}{2} \mathbf{S}(\omega)$, which follows from $\mathbf{R}^{T}(\tau)=$ $\mathbf{R}(-\tau)$.

Theorem 6. If $\mathbf{H}$ and $\mathbf{B}$ satisfy the basic conditions (Def. 价, then the extended power spectral density $\mathbf{G}(z)$ for the asymptotically stationary process $\mathbf{s}(t)$, defined in (2), is given by

$$
\mathbf{G}(z)=-\boldsymbol{\Sigma}^{-1}\left(\mathbf{H}^{T}-z \mathbf{I}\right)^{-1},
$$

provided $\operatorname{Re}\left[\mu_{l}+z\right]>0$ for $l=1, \ldots, n$.

Furthermore, the extended power spectral density $G(z)$ for the asymptotically stationary filter $\langle\mathbf{a}, \mathbf{s}(t)\rangle$ can be written as

$$
G(z)=\langle\mathbf{a}, \mathbf{G}(z) \mathbf{a}\rangle=-\sum_{l=1}^{n} \frac{\alpha_{l} \beta_{l}}{\mu_{l}+z},
$$

where $\alpha_{l}, \beta_{l}$ are defined in (85).

Proof. In Lemma 20, we showed that the autocorrelation function of the asymptotically stationary process $\mathbf{s}(t)$, in the limit $t \rightarrow \infty$, is given by $\mathbf{R}(\tau)=$ $\boldsymbol{\Sigma}^{-1} e^{\mathbf{H}^{T} \tau}$ where

$$
\boldsymbol{\Sigma}^{-1}=\lim _{t \rightarrow \infty} \int_{0}^{t} e^{\mathbf{H}(t-s)} \mathbf{B} e^{\mathbf{H}^{T}(t-s)} d s .
$$

From $\mathbf{R}(\tau)=\boldsymbol{\Sigma}^{-1} e^{\mathbf{H}^{T} \tau}$, we have

$$
\int_{0}^{\infty} \mathbf{R}(t) e^{-z t} d t=-\boldsymbol{\Sigma}^{-1}\left(\mathbf{H}^{T}-z \mathbf{I}\right)^{-1}
$$

assuming that $\operatorname{Re}\left[\mu_{l}+z\right]>0$ for $l=1, \ldots, n$ so that the integral converges. 
Since $\mathbf{a}$ is real, we can use (86) to write $\mathbf{a}=\sum_{k=1}^{n} \alpha_{k} \overline{\mathbf{v}}_{k}=\sum_{k=1}^{n} \bar{\alpha}_{k} \mathbf{v}_{k}$. Recall, we defined $\mathbf{v}_{l}$ so that $\mathbf{H}^{T} \mathbf{v}_{l}=-\bar{\mu}_{l} \mathbf{v}_{l}$, so we have $\left(\mathbf{H}^{T}-z \mathbf{I}\right)^{-1} \overline{\mathbf{v}}_{l}=\frac{-1}{\mu_{l}+z} \overline{\mathbf{v}}_{l}$ and $e^{\mathbf{H}^{T}(t-s)} \overline{\mathbf{v}}_{l}=e^{-\mu_{l}(t-s)} \overline{\mathbf{v}}_{l}$. Using these expressions along with (86), (82), and $\mathbf{B}=\mathbf{B}^{T}$, we compute

$$
\begin{aligned}
G(z) & =-\lim _{t \rightarrow \infty} \int_{0}^{t} \sum_{l, m=1}^{n} \bar{\alpha}_{m} \alpha_{l} \mathbf{v}_{m}^{T} e^{\mathbf{H}(t-s)} \mathbf{B} e^{\mathbf{H}^{T}(t-s)}\left(\mathbf{H}^{T}-z \mathbf{I}\right)^{-1} \overline{\mathbf{v}}_{l} d s \\
& =\sum_{l, m=1}^{n} \frac{\bar{\alpha}_{m} \alpha_{l}}{\mu_{l}+z}\left\langle\overline{\mathbf{v}}_{m}, \mathbf{B} \overline{\mathbf{v}}_{l}\right\rangle \lim _{t \rightarrow \infty} \int_{0}^{t} e^{-\left(\bar{\mu}_{m}+\mu_{l}\right)(t-s)} d s \\
& =\sum_{l, m=1}^{n} \frac{\bar{\alpha}_{m} \alpha_{l}}{\left(\bar{\mu}_{m}+\mu_{l}\right)\left(\mu_{l}+z\right)}\left\langle\mathbf{v}_{l}, \mathbf{B} \mathbf{v}_{m}\right\rangle=-\sum_{l=1}^{n} \frac{\alpha_{l} \beta_{l}}{\mu_{l}+z}
\end{aligned}
$$

Corollary 7. If $\mathbf{H}$ and $\mathbf{B}$ satisfy the basic conditions (Def. 4), then the power spectral density $S(\omega)$ of the asymptotically stationary filter $\langle\mathbf{a}, \mathbf{s}(t)\rangle$ is given by $S(\omega)=\langle\mathbf{a}, \mathbf{S}(\omega) \mathbf{a}\rangle$, where $\mathbf{S}(\omega)$ is the power spectral density of the asymptotically stationary process $\mathbf{s}(t)$, defined in (2), and

$$
\mathbf{S}(\omega)=\left(\mathbf{H}^{T}+i \omega \mathbf{I}\right)^{-1} \mathbf{B}\left(\mathbf{H}^{T}-i \omega \mathbf{I}\right)^{-1} .
$$

Proof. Using the expression for $\mathbf{G}$ in equation (80) we get

$$
\begin{aligned}
\mathbf{S}(\omega) & =2 \operatorname{Re}[\mathbf{G}(i \omega)]=\mathbf{G}(i \omega)+\mathbf{G}(i \omega)^{*} \\
& =-\boldsymbol{\Sigma}^{-1}\left(\mathbf{H}^{T}-i \omega \mathbf{I}\right)^{-1}-(\mathbf{H}+i \omega \mathbf{I})^{-1} \boldsymbol{\Sigma}^{-1} \\
& =-(\mathbf{H}+i \omega \mathbf{I})^{-1}\left((\mathbf{H}+i \omega \mathbf{I}) \boldsymbol{\Sigma}^{-1}+\boldsymbol{\Sigma}^{-1}\left(\mathbf{H}^{T}-i \omega \mathbf{I}\right)\right)\left(\mathbf{H}^{T}-i \omega \mathbf{I}\right)^{-1} \\
& =-(\mathbf{H}+i \omega \mathbf{I})^{-1}\left(\mathbf{H} \boldsymbol{\Sigma}^{-1}+\boldsymbol{\Sigma}^{-1} \mathbf{H}^{T}\right)\left(\mathbf{H}^{T}-i \omega \mathbf{I}\right)^{-1} \\
& =(\mathbf{H}+i \omega \mathbf{I})^{-1} \mathbf{B}\left(\mathbf{H}^{T}-i \omega \mathbf{I}\right)^{-1} .
\end{aligned}
$$

The asymptotic autocorrelation function for $\langle\mathbf{a}, \mathbf{s}(t)\rangle=\mathbf{a}^{T} \mathbf{s}(t)$ is given by $\left\langle\left\langle\mathbf{a}^{T} \mathbf{s}(t) \mathbf{s}^{T}(t+\tau) \mathbf{a}\right\rangle\right\rangle=\langle\mathbf{a}, \mathbf{R}(\tau) \mathbf{a}\rangle$. Hence $S(\omega)=\langle\mathbf{a}, \mathbf{S}(\omega) \mathbf{a}\rangle$.

\section{Appendix D: Supplementary Material for $\S 4$}

\section{Proof of Lemma 9}

Proof. We begin by defining

$$
\alpha_{k}=\left(\mathbf{U}^{T} \mathbf{a}\right)_{k}, \quad \beta_{k}=-\sum_{m=1}^{n} \frac{\bar{\alpha}_{m}}{\bar{\mu}_{m}+\mu_{k}}\left\langle\mathbf{v}_{k}, \mathbf{B} \mathbf{v}_{m}\right\rangle
$$

where $\mathbf{U}=\left[\mathbf{u}_{1}, \mathbf{u}_{2}, \ldots, \mathbf{u}_{n}\right]$. Recall, $\left\{\mathbf{u}_{j}\right\}$ are the eigenvectors of $\mathbf{H}$ and $\left\{\mathbf{v}_{j}\right\}$ are the normalized adjoint vectors. 
With $\mathbf{y}^{ \pm k}=\left(\mathbf{p}_{ \pm k}, \mathbf{q}_{ \pm k}, 0\right)^{T}$, from Lemma 3, we know the ladder operators can be written as

$$
\mathcal{L}_{k}=\mathbf{p}_{k} \cdot \nabla+\mathbf{q}_{k} \cdot \mathbf{s}, \quad k=-n, \ldots,-1,1, \ldots, n,
$$

with $\mathbf{p}_{ \pm k}$ and $\mathbf{q}_{ \pm k}$ given explicitly in the proof of Lemma 3. From these we see that for (37) to be satisfied we must have

$$
\sum_{k=1}^{n} \alpha_{k} \overline{\mathbf{v}}_{k}=\mathbf{a}, \quad \sum_{k=1}^{n} \beta_{k} \mathbf{u}_{k}=\sum_{j=1}^{n} \alpha_{j}\left(\mathbf{H}-\mu_{j} \mathbf{I}\right)^{-1} \mathbf{B} \overline{\mathbf{v}}_{j} .
$$

Hence, $\overline{\mathbf{V}} \boldsymbol{\alpha}=\mathbf{a}$, where $\mathbf{V}=\left[\mathbf{v}_{1}, \mathbf{v}_{2}, \ldots, \mathbf{v}_{n}\right]$. But $\mathbf{V}^{*} \mathbf{U}=\mathbf{I}$, so the first expression in (86) is equivalent to the definition of $\alpha_{k}$ in (85). Also, since the $\left\{\mathbf{u}_{k}\right\}$ are complete, and $\left(\left(\mathbf{H}-\mu_{j} \mathbf{I}\right)^{-1}\right)^{*} \mathbf{v}_{k}=-\left(\bar{\mu}_{k}+\bar{\mu}_{j}\right)^{-1} \mathbf{v}_{k}$ we conclude

$$
\begin{aligned}
\beta_{k} & =\left\langle\mathbf{v}_{k}, \sum_{j=1}^{n} \alpha_{j}\left(\mathbf{H}-\mu_{j} \mathbf{I}\right)^{-1} \mathbf{B} \overline{\mathbf{v}}_{j}\right\rangle \\
& =-\sum_{j=1}^{n} \frac{\alpha_{j}}{\mu_{k}+\mu_{j}}\left\langle\mathbf{v}_{k}, \mathbf{B} \overline{\mathbf{v}}_{j}\right\rangle=-\sum_{j=1}^{n} \frac{\bar{\alpha}_{j}}{\mu_{k}+\bar{\mu}_{j}}\left\langle\mathbf{v}_{k}, \mathbf{B} \mathbf{v}_{j}\right\rangle,
\end{aligned}
$$

where the last equality follows from a rearrangement of the sum over $j$, and the fact that the eigenvectors $\mathbf{v}_{j}$ and eigenvalues $\mu_{j}$ come in conjugate pairs. Thus, with $\alpha_{k}, \beta_{k}$ defined as in (85), the equations in (86) are satisfied, and therefore (37) holds.

\section{Proof of Theorem 3}

Proof. From Lemma 13 we have

$$
\begin{aligned}
\lambda_{2} & =-\left\langle\boldsymbol{\psi}_{1}, \sum_{k=1}^{n} \alpha_{k} \boldsymbol{\Gamma}_{1} \mathbf{c}_{k}\right\rangle=-\left\langle\boldsymbol{\psi}_{1}, \sum_{m=1}^{n} \sum_{j=1}^{J} \frac{\alpha_{m} \beta_{m}\left\langle\boldsymbol{\psi}_{j}, \boldsymbol{\Gamma}_{1} \boldsymbol{\phi}_{1}\right\rangle}{\nu_{1}-\nu_{j}+\mu_{m}} \boldsymbol{\Gamma}_{1} \boldsymbol{\phi}_{j}\right\rangle \\
& =-\sum_{j=1}^{J}\left\langle\boldsymbol{\psi}_{1}, \boldsymbol{\Gamma}_{1} \boldsymbol{\phi}_{j}\right\rangle\left\langle\boldsymbol{\psi}_{j}, \boldsymbol{\Gamma}_{1} \boldsymbol{\phi}_{1}\right\rangle \sum_{m=1}^{n} \frac{\alpha_{m} \beta_{m}}{\nu_{1}-\nu_{j}+\mu_{m}} \\
& =\sum_{j=1}^{J}\left\langle\boldsymbol{\psi}_{1}, \boldsymbol{\Gamma}_{1} \boldsymbol{\phi}_{j}\right\rangle\left\langle\boldsymbol{\psi}_{j}, \boldsymbol{\Gamma}_{1} \boldsymbol{\phi}_{1}\right\rangle G\left(\nu_{1}-\nu_{j}\right) .
\end{aligned}
$$

The last equality follows from (81).

\section{References}

[1] Ralph Abraham and Jerrold E. Marsden. Foundations of mechanics. Benjamin/Cummings Publishing Co. Inc. Advanced Book Program, Reading, Mass., 1978. Second edition, revised and enlarged, With the assistance of Tudor Raţiu and Richard Cushman. 
[2] Fred C. Adams and Anthony M. Bloch. Hill's equation with random forcing terms. SIAM J. Appl. Math., 68(4):947-980, 2008.

[3] Fred C. Adams and Anthony M. Bloch. Hill's equation with random forcing parameters: the limit of delta function barriers. J. Math. Phys., 50(7):073501, 20, 2009.

[4] Fred C. Adams and Anthony M. Bloch. Hill's equation with random forcing parameters: determination of growth rates through random matrices. $J$. Stat. Phys., 139(1):139-158, 2010.

[5] Ludwig Arnold. Stochastic differential equations as dynamical systems. In Realization and modelling in system theory (Amsterdam, 1989), volume 3 of Progr. Systems Control Theory, pages 489-495. Birkhäuser Boston, Boston, MA, 1990.

[6] V. I. Arnol'd. Geometrical methods in the theory of ordinary differential equations, volume 250 of Grundlehren der Mathematischen Wissenschaften [Fundamental Principles of Mathematical Sciences]. Springer-Verlag, New York, second edition, 1988. Translated from the Russian by Joseph Szücs [József M. Szücs].

[7] Søren Asmussen and Peter W. Glynn. Stochastic simulation: algorithms and analysis, volume 57 of Stochastic Modelling and Applied Probability. Springer, New York, 2007.

[8] Timothy Blass and L.A. Romero. On the stability of stochastically forced parametric oscillators. Sandia National Laboratories Report, SAND-20126980, 2012.

[9] R. V. Bobryk and A. Chrzeszczyk. Colored-noise-induced parametric resonance. Physica A, 316:225-232, 2002.

[10] David Carlson, B. N. Datta, and Hans Schneider. On the controllability of matrix pairs $(A, K)$ with $K$ positive semidefinite. SIAM J. Algebraic Discrete Methods, 5(3):346-350, 1984.

[11] P. A. M. Dirac. The Principles of Quantum Mechanics. Oxford, at the Clarendon Press, 1947. 3d ed.

[12] C. W. Gardiner. Handbook of stochastic methods, volume 13 of Springer Series in Synergetics. Springer-Verlag, Berlin, second edition, 1985. For physics, chemistry and the natural sciences.

[13] R.Z. Khasminski. Stochastic stability of differential equations. Kluwer Academic Pub, 1980.

[14] Peter E. Kloeden and Eckhard Platen. Numerical solution of stochastic differential equations, volume 23 of Applications of Mathematics (New York). Springer-Verlag, Berlin, 1992. 
[15] Horace Lamb. Hydrodynamics. Cambridge Mathematical Library. Cambridge University Press, Cambridge, sixth edition, 1993. With a foreword by R. A. Caflisch [Russel E. Caflisch].

[16] Hye Jin Lee, Changho Kim, Jae Gil Kim, and Eok Kyun Lee. A general scheme for studying the stochastic dynamics of a parametric oscillator driven by coloured noise. J. Phys. A, 37(3):647-656, 2004.

[17] Daniel Liberzon and Roger W. Brockett. Spectral analysis of Fokker-Planck and related operators arising from linear stochastic differential equations. SIAM J. Control Optim., 38(5):1453-1467, 2000.

[18] G. Metafune, D. Pallara, and E. Priola. Spectrum of Ornstein-Uhlenbeck operators in $L^{p}$ spaces with respect to invariant measures. J. Funct. Anal., 196(1):40-60, 2002.

[19] R. Repetto and V. Galletta. Finite amplitude Faraday waves induced by a random forcing. Physics of fluids, 14:4284, 2002.

[20] P.M.V. Résibois and M. De Leener. Classical kinetic theory of fluids. Wiley New York, 1977.

[21] H. Risken. The Fokker-Planck equation, volume 18 of Springer Series in Synergetics. Springer-Verlag, Berlin, second edition, 1989. Methods of solution and applications.

[22] R. Valéry Roy. Stochastic averaging of oscillators excited by colored Gaussian processes. Internat. J. Non-Linear Mech., 29(4):463-475, 1994.

[23] U. M. Titulaer. A systematic solution procedure for the Fokker-Planck equation of a Brownian particle in the high-friction case. Phys. A, 91(34):321-344, 1978.

[24] N. G. van Kampen. Stochastic processes in physics and chemistry, volume 888 of Lecture Notes in Mathematics. North-Holland Publishing Co., Amsterdam, 1981.

[25] Michael Wilkinson. Perturbation theory for a stochastic process with Ornstein-Uhlenbeck noise. J. Stat. Phys., 139(2):345-353, 2010. 